\title{
Renormalization of Feynman Integrals on the Lattice
}

\author{
Thomas Reisz ${ }^{\star}$ \\ Deutsches Elektronen-Synchrotron DESY, D-2000 Hamburg, Federal Republic of Germany
}

\begin{abstract}
A perturbative renormalization procedure is proposed which applies to massive field theories on a space-time lattice and is analogous to the BPHZ finite part prescription for continuum Feynman integrals. The renormalized perturbation theory is shown to be universal, i.e. under very natural assumptions the continuum limit exists and is independent of the details of the lattice action.
\end{abstract}

\section{Introduction}

In perturbation theory of a local quantum field theory there exist well-known renormalization procedures which remove ultraviolet divergencies. The BPHZ finite part prescription makes subtractions directly in the integrand of each Feynman integral in momentum space [1]. Divergencies of every subdiagram are subtracted by application of a Taylor operator in the external momenta of the subdiagram, which in position space is a local operation. The renormalized Feynman integral is defined in such a way that the ultraviolet (UV) divergence degrees of all subdiagrams are negative. There exists a power counting theorem due to Hahn and Zimmermann [2], which states the convergence of integrals having this property.

Unfortunately, these methods assume a rational structure of the Feynman integrands and hence do not apply to diagrams with a lattice cutoff. In this case, instead of being rational, the integrand is periodic with the Brillouin zone. Removing divergencies by subtraction of Taylor polynomials is very unnatural in a lattice description, and in fact such a procedure does not work, due to violation of periodicity. In a recent paper [3], we have proposed a lattice version of the power counting theorem of Hahn and Zimmermann by generalizing the well-known notion of a UV-divergence degree. Having such a theorem at our disposal, we are able to construct a generalization of the BPHZ finite part prescription which applies to diagrams with a lattice cutoff. Due to power counting conditions, the combinatorics of subtractions are given by the forest formula of Zimmermann [1]. As will be seen, the important modification consists in replacing Taylor operators 
by appropriate lattice subtraction operators. This class contains as a special case Taylor polynomials in "lattice momenta" $(\sin (q a) / a$ etc.), but is in fact much more general. Lattice diagrams renormalized in such a way are convergent when the cutoff is removed. The limit itself is universal, i.e. it does not depend on the lattice action chosen, and it is given by continuum Feynman integrals which are renormalized by the continuum BPHZ prescription.

To avoid infrared singularities, we have assumed that all fields are massive. At first we introduce in Sect. 2 a couple of notations concerning Feynman diagrams and integrals on the lattice. In Sect. 3 the important notion of a lattice subtraction operator is introduced, and its most important properties are given. The lattice version of the BPHZ renormalization is defined in Sect. 4. It is shown that subtractions, if appropriately chosen, can be written as counterterm contributions to the lattice action. General conditions are stated which guarantee power counting renormalizability of a lattice field theory. In Sect. 5 the convergence proof of the renormalization scheme is given, using Zimmermann's method of "complete forests." For simplicity, all formulas are written for scalar fields only. In Sect. 6, the modifications necessary to include fields carrying internal symmetries and spin are briefly described.

Throughout this paper, we will use the notations of [3]. These are listed for completeness in Appendix A, and there are also given some general examples of subtraction operators. The Appendices B and C contain some lemmas used in the text.

\section{Feynman Diagrams and Integrals}

2.1. Topology of Feynman Diagrams. We define topological notions of Feynman diagrams and Feynman integrals. In part, our notations are those of Zimmermann [1] and Nakanishi [5].

A (Feynman) diagram or (Feynman) graph $\Gamma$ is a structure

$$
\Gamma=\left(\mathscr{L}_{\Gamma}, \mathscr{E}_{\Gamma}, \mathscr{B}_{\Gamma}, \phi_{\Gamma}, \psi_{\Gamma}\right)
$$

having the following properties: $\mathscr{L}_{\Gamma}, \mathscr{E}_{\Gamma}, \mathscr{B}_{\Gamma}$ are mutually disjoint sets,

$$
\begin{array}{cl}
\mathscr{L}_{\Gamma}=\left\{L_{1}, \ldots, L_{P(\Gamma)}\right\} & \text { internal lines of } \Gamma, \\
\mathscr{E}_{\Gamma}=\left\{E_{1}, \ldots, E_{N(\Gamma)}\right\} & \text { external lines of } \Gamma, \\
\mathscr{B}_{\Gamma}=\left\{B_{1}, \ldots, B_{M(\Gamma)}\right\} & \text { vertices of } \Gamma .
\end{array}
$$

$\phi_{\Gamma}$ and $\psi_{\Gamma}$ are the incidence relations of $\Gamma$, i.e. they are mappings of the form

$$
\phi_{\Gamma}: \mathscr{L}_{\Gamma} \rightarrow \mathscr{B}_{\Gamma} \times \mathscr{B}_{\Gamma}, \quad \phi_{\Gamma}(L)=\left(A_{L}, B_{L}\right), \quad \psi_{\Gamma}: \mathscr{E}_{\Gamma} \rightarrow \mathscr{B}_{\Gamma}, \quad \psi_{\Gamma}(E)=A_{E} .
$$

$A_{L}$ is called the outgoing endpoint and $B_{L}$ the ingoing endpoint of $L \in \mathscr{L}_{\Gamma}$. Both $A_{L}$ and $B_{L}$ are called endpoints of $L$. If $A_{L}=B_{L}$, then $L$ is called a loop line. If $A \in \mathscr{B}_{\Gamma}$ and there exists an $E \in \mathscr{E}_{\Gamma}$ such that $\psi_{\Gamma}(E)=A$, then $A$ is called an external vertex of $\Gamma$. Otherwise, $A$ is called an internal vertex of $\Gamma$.

Let $\gamma$ and $\Gamma$ be diagrams. $\gamma$ is called a subdiagram (or subgraph) of $\Gamma$ (Fig. 1), if

1.

$$
\mathscr{L}_{\gamma} \subseteq \mathscr{L}_{\Gamma}, \quad \mathscr{B}_{\gamma} \subseteq \mathscr{B}_{\Gamma}, \quad \phi_{\gamma}=\phi_{\Gamma} / \mathscr{L}_{\gamma}
$$



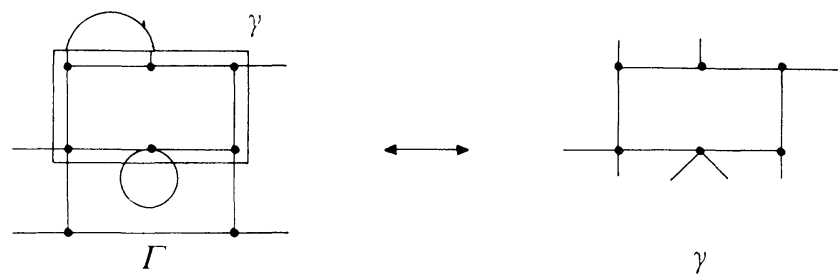

Fig. 1. A diagram $\gamma$ as a subdiagram of $\Gamma$

(i.e., $\phi_{\gamma}$ is the restriction of $\phi_{\Gamma}$ onto $\mathscr{L}_{\gamma}$. Especially, $\left.\phi_{\gamma}\left(\mathscr{L}_{\gamma}\right) \subseteq \mathscr{B}_{\gamma} \times \mathscr{B}_{\gamma}\right)$.

2. Every $E \in \mathscr{E}_{\Gamma}$ satisfying $\psi_{\Gamma}(E) \in \mathscr{B}_{\gamma}$ is in $\mathscr{E}_{\gamma}$, and

$$
\psi_{\gamma}(E)=\psi_{\Gamma}(E)
$$

The set of these lines is denoted by $\mathscr{E}_{\gamma(\Gamma)}^{\text {ext }}$.

3. Every $E \in \mathscr{L}_{\Gamma} \backslash \mathscr{L}_{\gamma}$, which has exactly one endpoint $B_{E}$ in $\mathscr{B}_{\gamma}$ under $\phi_{\Gamma}$, is in $\mathscr{E}_{\gamma}$, and

$$
\psi_{\gamma}(E)=B_{E} .
$$

The set of these $E$ is denoted by $\mathscr{E} \gamma(\Gamma)$.

4. The remaining external lines in $\mathscr{E}_{\gamma}$ (i.e. those which are not in $\mathscr{E}_{\gamma(\Gamma)}^{\text {ext }}$ or $\mathscr{E}_{\gamma(\Gamma)}^{\text {int }}$ ) are obtained in the obvious way by cutting in to the lines $L \in \mathscr{L}_{\Gamma} \backslash \mathscr{L}_{\text {}}$, which have both endpoints in $\mathscr{B}_{\gamma}$. Every such line is called a loop line of $\Gamma$ relative to $\gamma$. Furthermore, we set $\mathscr{E}_{\gamma(\Gamma)}^{\text {loop }}=\mathscr{E}_{\gamma} \backslash\left(\mathscr{E}_{\gamma(\Gamma)}^{\operatorname{sint}} \cup \mathscr{E}_{\gamma(\Gamma)}^{\text {ext }}\right)$.

If every $B \in \mathscr{B}_{\gamma}$ is an endpoint of at least one line in $\mathscr{L}_{\gamma}$, then $\gamma$ is called the subdiagram spanned by $\mathscr{L}_{\gamma}$ (This is the definition of a subgraph in the sense of Zimmermann [1].)

A diagram $\Gamma$ is said to be connected, if for all pairs of vertices $B_{1}, B_{2} \in \mathscr{B}_{\Gamma}$, $B_{1} \neq B_{2}$ there is a set of lines

$$
\left\{L_{1}, \ldots, L_{c}\right\} \subseteq \mathscr{L}_{\Gamma}
$$

such that $B_{1}$ is an endpoint of $L_{1}, B_{2}$ an endpoint of $L_{c}$, and for all $i=1, \ldots, c-1$ the lines $L_{i}$ and $L_{i+1}$ have one endpoint in common. A subset $\mathscr{L} \subseteq \mathscr{L}_{\Gamma}$ of lines is called a connected set of lines, if the subdiagram of $\Gamma$ spanned by $\mathscr{L}$ is connected. Two connected subgraphs $\gamma_{1}, \gamma_{2}$ are said to be disjoint, if they have neither vertices nor internal lines in common. A graph is disconnected, if it is not connected.

A graph $\Gamma$ is called 1PI (one-particle irreducible), if $\mathscr{L}_{\Gamma} \neq \emptyset$, and there exists no line $L \in \mathscr{L}_{\Gamma}$ such that the diagram spanned by $\mathscr{L}_{\Gamma} \backslash\{L\}$ is disconnected.

Let $\Gamma$ be a Feynman diagram and $\gamma$ a connected subdiagram of $\Gamma$. The reduced diagram

$$
\Gamma / \gamma=\left(\mathscr{L}_{\Gamma / \gamma}, \mathscr{E}_{\Gamma / \gamma}, \mathscr{B}_{\Gamma / \gamma}, \phi_{\Gamma / \gamma}, \psi_{\Gamma / \gamma}\right)
$$

is defined as follows (Fig. 2):

$$
\mathscr{L}_{\Gamma / \gamma}=\mathscr{L}_{\Gamma} \backslash \mathscr{L}_{\gamma}, \quad \mathscr{E}_{\Gamma / \gamma}=\mathscr{E}_{\Gamma}, \quad \mathscr{B}_{\Gamma / \gamma}=\mathscr{B}_{\Gamma} \backslash \mathscr{B}_{\gamma} \cup\{\bar{B}\} .
$$

$\bar{B} \notin \mathscr{B}_{\Gamma}$ is called a reduced vertex. For every $L \in \mathscr{L}_{\Gamma / \gamma}, \phi_{\Gamma}(L)=\left(A_{L}, B_{L}\right)$, 


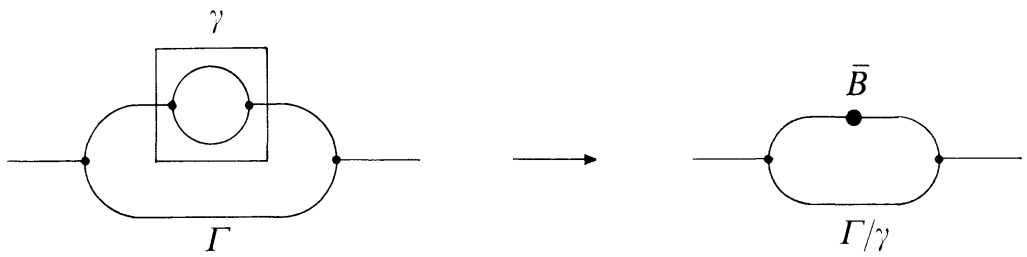

Fig. 2. A reduce diagram $\Gamma / \gamma$

$$
\phi_{\Gamma / \gamma}(L)=\left\{\begin{array}{lll}
\left(A_{L}, B_{L}\right) & \text { if } & A_{L}, B_{L} \notin \mathscr{B}_{\gamma} \\
\left(A_{L}, \bar{B}\right) & \text { if } & A_{L} \notin \mathscr{B}_{\gamma}, B_{L} \in \mathscr{B}_{\gamma} \\
\left(\bar{B}, B_{L}\right) & \text { if } & A_{L} \in \mathscr{B}_{\gamma}, B_{L} \notin \mathscr{B}_{\gamma} \\
(\bar{B}, \bar{B}) & \text { if } & A_{L}, B_{L} \in \mathscr{B}_{\gamma}
\end{array}\right.
$$

and for every $E \in \mathscr{E}_{\Gamma / \gamma}, \psi_{\Gamma}(E)=B_{E}$,

$$
\psi_{\Gamma / \gamma}(E)=\left\{\begin{array}{lll}
B_{E} & \text { if } & B_{E} \notin \mathscr{B}_{\gamma} \\
\bar{B} & \text { if } & B_{E} \in \mathscr{B}_{\gamma}
\end{array} .\right.
$$

By induction, a reduced graph can be defined for mutually disjoint, connected subdiagrams $\gamma_{1}, \ldots, \gamma_{c}$ of $\Gamma$. To every $\gamma_{i}$ there corresponds exactly one reduced vertex $\bar{B}_{i}$ in $\Gamma / \gamma_{1} \cdots \gamma_{c}$.

Let $B \in \mathscr{B}_{\Gamma}$ and $\mathscr{L} \subseteq \mathscr{L}_{\Gamma}$. Then the line number $D(B, \mathscr{L})$ of $\mathscr{L}$ with respect to $B$ is defined as the sum of the number of lines in $\mathscr{L}$, having $B$ as its outgoing endpoint, and the number of lines in $\mathscr{L}$, having $B$ as ingoing endpoint. Especially, loop lines are counted twice.

Let $B_{1}, B_{2} \in \mathscr{B}_{\Gamma}, B_{1} \neq B_{2} . \mathscr{L} \subseteq \mathscr{L}_{\Gamma}$ is called a path between $B_{1}$ and $B_{2}$, if $\mathscr{L}$ is connected, $D\left(B_{1}, \mathscr{L}\right)=D\left(B_{2}, \mathscr{L}\right)=1$ and $D(B, \mathscr{L}) \in\{0,2\}$ for all other $B \in \mathscr{B}_{\Gamma}$. $\mathscr{C} \subseteq \mathscr{L}_{\Gamma}$ is called a loop in $\Gamma$ if $\mathscr{C}$ is connected and $D(B, \mathscr{C}) \in\{0,2\}$ for all $B \in \mathscr{B}_{\Gamma}$.

Let $\Gamma$ be a connected diagram. A tree in $\Gamma$ is a maximal set $\mathscr{T} \subseteq \mathscr{L}_{\Gamma}$ of lines containing no loop. $\mathscr{T}^{*}=\mathscr{L}_{\Gamma} \backslash \mathscr{T}$ is called the chord set of $\mathscr{T}$ in $\Gamma$. $\mathscr{T}^{*}$ has $m(\Gamma)=P(\Gamma)-M(\Gamma)+1$ lines, where $P(\Gamma)$ is the number of internal lines of $\Gamma$ and $M(\Gamma)$ is the number of vertices of $\Gamma . m(\Gamma)$ is called the number of loops in $\Gamma[5]$. It is independent of the tree chosen. $\mathscr{T}^{*}$ contains all loop lines.

Lemma 2.1 [5]. A tree $\mathscr{T}$ in $\Gamma$ has the following properties:

a. $D(B, \mathscr{T}) \neq \emptyset$ for each $B \in \mathscr{B}_{\Gamma}$.

b. $\mathscr{T}$ is connected.

c. For any $B_{1}, B_{2} \in \mathscr{B}_{\Gamma}, B_{1} \neq B_{2}$, there is a unique path $\mathscr{P} \subseteq \mathscr{T}$ between $B_{1}$ and $B_{2}$.

d. The number of lines in $\mathscr{T}$ is $M(\Gamma)-1$.

2.2. Momentum Distributions. Having defined the topology of Feynman diagrams, we will now discuss momentum distributions and the structure of Feynman integrals on an infinite space-time lattice. First of all we define incidence numbers.

Let $\Gamma$ be a Feynman diagram and $L \in \mathscr{L}_{\Gamma}, \phi_{\Gamma}(L)=\left(B_{1}, B_{2}\right), B_{1} \neq B_{2}$. Then, for every $B \in \mathscr{B}_{\Gamma}$, we define incidence numbers by

$$
\left[B_{1}: L\right]=-1, \quad\left[B_{2}: L\right]=+1, \quad[B: L]=0 \quad \text { if } \quad B \neq B_{1}, B_{2} .
$$


If $B_{1}=B_{2}$, then $L$ is a loop line, and we set

$$
[B: L]=0 \text { for all } B \in \mathscr{B}_{\Gamma} .
$$

For every external line $E \in \mathscr{E}_{\Gamma}, \psi_{\Gamma}(E)=B_{1} \in \mathscr{B}_{\Gamma}$, we set

$$
[B: L]=0 \quad \text { if } \quad B \neq B_{1}, \quad\left[B_{1}: L\right]=+1 \quad \text { or }-1 .
$$

The choice between +1 and -1 is arbitrary. If $\gamma$ is a subdiagram of $\Gamma$, we define induced incidence numbers of $\gamma$ by

$$
\begin{array}{lll}
{[B: L]_{\gamma}=[B: L]} & \text { if } & L \in \mathscr{L}_{\gamma} \cup \mathscr{E}_{\gamma(\Gamma)}^{\operatorname{int}} \cup \mathscr{E}_{\gamma(\Gamma)}^{\text {ext }}, \\
{[B: L]_{\gamma}=+1 \text { or }-1} & \text { if } L \in \mathscr{E}_{\gamma(\Gamma)}^{\text {lop }} .
\end{array}
$$

Again, the choice between -1 and +1 is arbitrary.

A momentum distribution in $\Gamma$ is a map

$$
\mathscr{M}: \mathscr{L}_{\Gamma} \cup \mathscr{E}_{\Gamma} \rightarrow \mathbf{R}^{4}, \quad L \rightarrow l_{L},
$$

so that

$$
\sum_{L \in \mathscr{L}_{\Gamma} \cup \mathscr{E}_{\Gamma}}[B: L] l_{L}=0 \text { for all } B \in \mathscr{B}_{\Gamma}
$$

This means momentum conservation at each vertex. $l_{L}$ flows from the outgoing endpoint $B([B: L]=-1)$ to the ingoing endpoint $B^{\prime}$ of $L\left(\left[B^{\prime}: L\right]=+1\right)$. The momenta of loop lines are not restricted by (2-2).

Let $\Gamma$ be a connected diagram. Then the only relations between the incidence numbers are

$$
\sum_{B \in B_{\Gamma}}[B: L]=0 \text { for all } L \in \mathscr{L}_{\Gamma} .
$$

For every external line $E \in \mathscr{E}_{\Gamma}$ set

$$
q_{E}=l_{E} \sum_{B \in B_{\Gamma}}[B: E]
$$

(the sum contains exactly one element). $q_{E}$ are called external momenta of $\Gamma$. They are flowing into the diagram. If (2-2) is summed over all vertices $B \in \mathscr{B}_{\Gamma}$, we get conservation of the overall momentum

$$
\sum_{E \in \mathscr{\delta}_{\Gamma}} q_{E}=0
$$

We shall assume all $q_{E}, E \in \mathscr{E}_{\Gamma}$, are given momenta, such that (2-4) is satisfied. Then $(2-2)$ is a system of equations for the line momenta $l_{L}, L \in \mathscr{L}_{\Gamma}$ :

$$
\sum_{L \in \mathscr{L}_{\Gamma}}[B: L] l_{L}+\sum_{E \in \mathscr{E}_{\Gamma}}|[B: L]| q_{E}=0 \text { for all } B \in \mathscr{B}_{\Gamma}
$$

The system (2-5) is solvable, the matrix

$$
\mathscr{A}_{\Gamma}=\left([B: L] \mid B \in \mathscr{B}_{\Gamma}, L \in \mathscr{L}_{\Gamma}\right)
$$

having rank $\mathscr{A}_{\Gamma}=M(\Gamma)-1$, and because of (2-4).

In what follows let $\Gamma$ be a 1PI diagram. We shall define a partition of line momenta into internal and external momenta. At first, we choose a basis of the external momenta. Fix $E_{0} \in \mathscr{E}_{\Gamma}$. Then define 


$$
q=q^{\Gamma}=\left(q_{E} \mid E \in \mathscr{E}_{\Gamma}, E \neq E_{0}\right) .
$$

$q_{E_{0}}$ is given by momentum conservation. For every $L \in \mathscr{L}_{\Gamma}$ we write $l_{L}=k_{L}+q_{L}$, defined as follows:

1. We choose an arbitrary tree $\mathscr{T}$ in $\Gamma$. For $L_{1}, \ldots, L_{m} \in \mathscr{T}^{*}, m$ being the number of loops in $\Gamma$, we define

and set

$$
l_{L_{l}}=k_{i}, \quad \text { i.e., } \quad q_{L_{l}}=0, \quad i=1, \ldots, m
$$

$$
k=k^{\Gamma}=\left(k_{1}, \ldots, k_{m}\right)
$$

Remember that $m$ is independent of the choice of a tree. $\left(k_{1}, \ldots, k_{m}\right)$ is called a basis of the internal momenta of $\Gamma$ for the following reason:

The endpoints of any $L \in \mathscr{T}^{*}$ can be connected by a unique path $\mathscr{P}_{L}$ in $\mathscr{T}$. $\mathscr{P}_{L} \cup\{L\}$ is a loop in $\Gamma$ (if $L$ is a loop line, then $\mathscr{P}_{L}=\emptyset$, and the loop is $\{L\}$ ). For every $L \in \mathscr{T}$ the internal line momenta $k_{L}$ are defined as the unique solution of

$$
\sum_{L \in \mathscr{L}_{\Gamma}}[B: L] k_{L}=0 \text { for all } B \in \mathscr{B}_{\Gamma}
$$

and are of the form

$$
k_{L}=k_{L}(k)=\sum_{j=1}^{m}\left(C_{L}\right)_{j} k_{j}, \quad\left(C_{L}\right)_{j} \in \mathbf{Z} \text { for all } L \in \mathscr{L}_{\Gamma} .
$$

This is the general homogeneous solution of (2-5) in a form dependent on a chosen tree. We remark in passing that this shows that the line momenta are natural in the sense of [3].

2. External line momenta $q_{L}=q_{L}(q), L \in \mathscr{T}$, are now defined as the unique solution of

$$
\sum_{L \in \mathscr{T}}[B: L] q_{L}+\sum_{E \in \mathscr{E}_{r}}|[B: E]| q_{E}(q)=0 \text { for all } B \in \mathscr{B}_{\Gamma} .
$$

In summary,

$$
l_{L}(k, q)=k_{L}(k)+q_{L}(q) \text { for all } L \in \mathscr{L}_{\Gamma} .
$$

We now define an (unrenormalized) Feynman integral of $\Gamma$ by

$$
\hat{\mathscr{J}}_{\Gamma}(q ; \mu, a)=\int_{-\pi / a}^{\pi / a} d^{4} k_{1} \cdots d^{4} k_{m} \hat{I}_{\Gamma}(k, q ; \mu, a),
$$

where $m$ is the number of loops in $\Gamma$. The unrenormalized Feynman integrand $\hat{I}_{\Gamma}$ is

$$
\hat{I}_{\Gamma}(k, q ; \mu, a)=\prod_{B \in \mathscr{B}_{\Gamma}} \hat{V}_{B}\left(\left\{l_{L}\right\}_{B} ; \mu, a\right) \cdot \prod_{L \in \mathscr{L}_{\Gamma}} \hat{\Delta}_{L}\left(l_{L} ; \mu, a\right) .
$$

For every $L \in \mathscr{L}_{\Gamma}$ the propagators are of the form

$$
\hat{\Delta}_{L}\left(l_{L} ; \mu, a\right)=\frac{\hat{P}_{L}\left(l_{L} ; \mu, a\right)}{\prod_{j=1}^{n(L)}\left[e_{L j}\left(l_{L} ; a\right)+\mu_{L j}^{2}\right]},
$$

where $n(L) \in \mathbf{N}$ and

$$
\hat{P}_{L}\left(l_{L} ; \mu, a\right)=\sum_{(i)} P^{(i)}(\mu) V_{(i)}\left(l_{L} ; a\right) .
$$

The ( $L$-dependent) sum is finite, $P^{(i)}$ are polynomials in the masses $\mu$ and 
$V_{(i)} \in \mathscr{C}_{m_{i}}^{c}, m_{i} \in \mathbf{Z}$, periodic in $l_{L}$ with the Brillouin zone (BZ) $[-\pi / a, \pi / a]^{4}$. The function classes $\mathscr{C}_{m}^{c}$ are define in [3] (see Appendix A). In most applications the sum contains one term only. Furthermore,

$$
\begin{gathered}
\mu_{L j}^{2}>0, \\
e_{L j} \in \mathscr{C}_{2}^{c}, \quad e_{L j}\left(l_{L} ; a\right)=\frac{1}{a^{2}} \eta_{L j}\left(l_{L} a\right), \\
\eta_{L j}\left(l_{L} a \neq 0\right)>0 \quad \text { if } l_{L} \in[-\pi / a, \pi / a]^{4}, \\
\eta_{L j} \text { is }(2 \pi / a) \text {-periodic in every component of } l_{L}, \\
\lim _{a \rightarrow 0} e_{L j}\left(l_{L} ; a\right)=l_{L}^{2} .
\end{gathered}
$$

This means that $\hat{\Delta}_{L}$ belongs to the class $\mathscr{F}$ of functions which is defined in [3]. Especially, the propagators have only one pole in the BZ. For every vertex $B \in \mathscr{B}_{\Gamma}$ the functions $\hat{V}_{B} \in \mathscr{C}^{c}$ are of the form (2-12) in variables $\left\{l_{L}\right\}_{B}$, which are the momenta of the lines $L \in \mathscr{L}_{\Gamma} \cup \mathscr{E}_{\Gamma}$ having $B$ as one of its endpoints. For uniqueness, we consider $\hat{V}_{B}$ to be a function of the momenta flowing into the vertex $B$. Furthermore, we always assume $\hat{V}_{B}$ to be periodic with the $\mathrm{BZ}$, in all momentum variables. As discussed above, all line momenta $l_{L}$ are written as

$$
l_{L}(k, q)=k_{L}(k)+q_{L}(q) \text { for all } L \in \mathscr{L}_{\Gamma} \text {. }
$$

The integrand $\hat{I}_{\Gamma}$ belongs to the function class $\mathscr{F}$.

Next, we define internal and external momenta of a 1PI subgraph $\gamma$ of $\Gamma$. For every external line $E \in \mathscr{E}_{\gamma}$ let $q_{E}^{\gamma}$ be a momentum flowing into the diagram, and such that

$$
\sum_{E \in \mathscr{E}_{\gamma}} q_{E}^{\gamma}=0
$$

Fixing $E_{0 \gamma} \in \mathscr{E}_{\gamma}$, we get a basis of the external momenta of $\gamma$,

$$
q^{\gamma}=\left(q_{E}^{\gamma} \mid E \in \mathscr{E}_{\gamma}, E \neq E_{0 \gamma}\right) .
$$

We define a momentum distribution in the diagram $\gamma$ :

$$
\mathscr{M}_{\gamma}: \mathscr{L}_{\gamma} \cup \mathscr{E}_{\gamma} \rightarrow \mathbf{R}^{4}, \quad L \rightarrow l_{L}^{\gamma} \quad \text { for } \quad L \in \mathscr{L}_{\gamma}, \quad E \rightarrow q_{E}^{\gamma} \text { for } E \in \mathscr{E}_{\gamma},
$$

such that

$$
\sum_{L \in \mathscr{L}_{\gamma}}[B: L]_{\gamma} l_{L}^{\gamma}+\sum_{E \in \mathscr{E}_{\gamma}}\left|[B: E]_{\gamma}\right| q_{E}^{\gamma}\left(q^{\gamma}\right)=0 \text { for all } B \in \mathscr{B}_{\gamma},
$$

where now the line momenta $l_{L}^{\gamma}$

$$
l_{L}^{\gamma}\left(k^{\gamma}, q^{\gamma}\right)=k_{L}^{\gamma}\left(k^{\gamma}\right)+q_{L}^{\gamma}\left(q^{\gamma}\right)
$$

are partitioned as follows:

1. For a tree $\mathscr{T}$ in $\Gamma, \mathscr{T} \cap \mathscr{L}_{\gamma}$ is not necessarily a tree in $\gamma$. But it can always be completed to such a tree $\mathscr{T}_{\gamma}$. The chord set $\mathscr{T}_{\gamma}^{*}=\mathscr{L}_{\gamma} \backslash \mathscr{T}_{\gamma}$ in $\gamma$ contains $m(\gamma)$ lines, where $m(\gamma)$ is the number of loops in $\gamma$. For these lines $L_{i}$ we define

$$
l_{L_{i}}^{\gamma}=k_{i}^{\gamma}, \quad i=1, \ldots, m(\gamma)
$$


and we set

$$
k^{\gamma}=\left(k_{1}^{\gamma}, \ldots, k_{m(\gamma)}^{\gamma}\right)
$$

as a basis of the internal momenta of $\gamma$. Note that for every $i=1, \ldots, m(\gamma)$ there is a $S(i) \in\{1, \ldots, m(\Gamma)\}$ such that

$$
k_{i}^{\gamma}=k_{S(i)},
$$

and $\{S(i) \mid i=1, \ldots, m(\gamma)\}$ contains $m(\gamma)$ elements. For every $L \in \mathscr{T}_{\gamma}$ we define $k_{L}^{\gamma}$ as the solution of

so that

$$
\sum_{L \in \mathscr{L}_{\gamma}}[B: L]_{\gamma} k_{L}^{\gamma}=0 \text { for all } B \in \mathscr{B}_{\gamma}
$$

$$
k_{L}^{\gamma}=k_{L}^{\gamma}\left(k^{\gamma}\right) \text { for all } L \in \mathscr{L}_{\gamma} .
$$

By (2-17), (2-18), (2-19), the set

$$
\bigcup_{\gamma}\left\{k_{L}^{\gamma}\left(k^{\gamma}\right) \mid L \in \mathscr{L}_{\gamma}\right\}
$$

is natural in the sense of [3], where the union is over all 1PI subgraphs $\gamma$ of $\Gamma$ ( $\Gamma$ included).

2. $q_{L}^{\gamma}\left(q^{\gamma}\right), L \in \mathscr{T}_{\gamma}$, are defined as the unique solution of

$$
\sum_{L \in \mathscr{T}_{\gamma}}[B: L]_{\gamma} q_{L}^{\gamma}+\sum_{E \in \mathscr{E}_{\gamma}}\left|[B: E]_{\gamma}\right| q_{E}^{\gamma}\left(q^{\gamma}\right)=0 \text { for all } B \in \mathscr{B}_{\gamma} .
$$

Having defined internal and external momenta $k^{\gamma}, q^{\gamma}$, of $\gamma$, we will later need their relation to $k, q$. Set $k^{\Gamma}=k, q^{\Gamma}=q$ and $l_{L}^{\Gamma}=l_{L}$ for $L \in \mathscr{L}_{\Gamma}$. We define $k^{\gamma}\left(k^{\Gamma}\right)$ by $(2-17)$ and $q^{\gamma}\left(k^{\Gamma}, q^{\Gamma}\right)$ by identifying every $q_{E}^{\gamma}, E \in \mathscr{E}_{\gamma}$, with the momentum $\pm l_{L}^{\Gamma}$ of the line $L \in \mathscr{L}_{\Gamma} \cup \mathscr{E}_{\Gamma}$ which corresponds to $E$ by considering $\gamma$ as a subdiagram of $\Gamma$. The sign is determined by the condition that $q_{E}^{\gamma}$ always flows into the subdiagram $\gamma$. It will be convenient to represent this map by a linear substitution operator $S_{\Gamma}[1]$ :

$$
\begin{aligned}
S_{\Gamma}: k^{\gamma} & \left.\rightarrow k^{\gamma}\left(k^{\Gamma}\right) \quad \text { (independent of } q !\right) \\
q^{\gamma} & \rightarrow q^{\gamma}\left(k^{\Gamma}, q^{\Gamma}\right) .
\end{aligned}
$$

Note that the $k^{\Gamma}$-dependence of $q^{\gamma}$ is only by the explicit $k^{\Gamma}$-dependence of $q_{E}^{\gamma}\left(k^{\Gamma}, q^{\Gamma}\right)$ of external lines of $\gamma$. Furthermore, $k^{\gamma}$ is independent of the external momenta $q$ of $\Gamma$. From (2-5) and (2-15) it follows that

$$
l_{L}^{\gamma}\left(k^{\gamma}\left(k^{\Gamma}\right), q^{\gamma}\left(k^{\Gamma}, q^{\Gamma}\right)\right) \equiv l_{L}^{\Gamma}\left(k^{\Gamma}, q^{\Gamma}\right) \text { for all } L \in \mathscr{L}_{\gamma} .
$$

The definition of internal and external momenta can be generalized to an arbitrary subdiagram $\tau$ of $\gamma$ ( $\mu$ of $\tau$ etc.). In the formulation above, we have only to substitute $\gamma \rightarrow \tau, \Gamma \rightarrow \gamma$, and we get linear functions

$$
\begin{aligned}
l_{L}^{\tau}\left(k^{\tau}, q^{\tau}\right) & =k_{L}^{\tau}\left(k^{\tau}\right)+q_{L}^{\tau}\left(q^{\tau}\right)=l_{L}^{\gamma}\left(k^{\gamma}, q^{\gamma}\right) \text { for all } L \in \mathscr{L}_{\tau}, \\
k^{\tau} & =\left(k_{1}^{\tau}, \ldots, k_{m(\tau)}^{\tau}\right) \\
q^{\tau} & =\left(q_{E}^{\tau} \mid E \in \mathscr{E}_{\tau}, E \neq E_{0 \tau}\right),
\end{aligned}
$$

where $m(\tau)$ is the number of loops in $\tau$ and $E_{0 \tau} \in \mathscr{E}_{\tau}$ is a fixed external line of $\tau$. $k^{\tau}, q^{\tau}$ are functions of $k^{\gamma}, q^{\gamma}$ via $S_{\gamma}$ :

$$
S_{\gamma}: k^{\tau}=k^{\tau}\left(k^{\gamma}\right), \quad q^{\tau}=q^{\tau}\left(k^{\gamma}, q^{\gamma}\right),
$$


where again $k^{\gamma}$-dependence of $q^{\tau}$ via $S_{\gamma}$ is only by explicit $k^{\gamma}$-dependence of $q_{E}^{\tau}\left(k^{\gamma}, q^{\gamma}\right)$ of external lines of $\tau$. Applied to a function $\hat{f}$ in $k^{\tau}, q^{\tau}$

$$
S_{\gamma} \hat{f}\left(k^{\tau}, q^{\tau} ; a\right)=\widehat{f}\left(k^{\tau}\left(k^{\gamma}\right), q^{\tau}\left(k^{\gamma}, q^{\gamma}\right) ; a\right) .
$$

We will need a definition of internal and external momenta for arbitrary sequences of ordered 1PI subdiagrams. It is important to determine internal momenta of a subdiagram $\gamma$ always in the same way, independent of other diagrams in the sequence, i.e., always the same chord set $\mathscr{T}_{\gamma}^{*}$ to define $k^{\gamma}=\left(k_{1}^{\gamma}, \ldots, k_{m(\gamma)}^{\gamma}\right)$ has to be chosen. This must be done in such a way that $(2-22)$ is always satisfied. For arbitrary 1PI subgraphs $\gamma, \tau$ of $\Gamma, \tau$ being a subgraph of $\gamma$, we choose

$$
\mathscr{T}_{\tau}^{*} \subseteq \mathscr{T}_{\gamma}^{*} \cap \mathscr{L}_{\gamma},
$$

i.e. all lines in the chord set of $\tau$ are also in the chord set of $\gamma$. This can always be achieved. Let $P$ be the number of lines in a connected graph $\Gamma$ and $\mathscr{L}_{\Gamma}=$ $\left\{L_{1}, \ldots, L_{p}\right\}$. For every connected subdiagram $\gamma$ of $\Gamma$ we define $\mathscr{T}_{\gamma}^{*} \subseteq \mathscr{L}_{\gamma}$ as follows: $L_{j} \in \mathscr{T}_{\gamma}^{*}$ if and only if $\mathscr{L}_{\gamma} \backslash\left\{L_{1}, \ldots, L_{j-1}\right\}$ contains a loop $\mathscr{C}$ such that $\mathscr{C} \supseteq\left\{L_{j}\right\}$. In Appendix B it is shown that $\mathscr{T}_{\gamma}^{*}$ is indeed a chord set in $\gamma$ (i.e., $\mathscr{T}_{\gamma}=\mathscr{L}_{\gamma} \backslash \mathscr{T}_{\gamma}^{*}$ is a tree in $\gamma$ ). Apparently, (2-24) is a direct consequence of this construction. Also, an arbitrary chord set $\mathscr{T}_{\Gamma}^{*}$ of $\Gamma$ can be achieved by this way. For, if $m$ is the number of loops in $\Gamma$ and $\mathscr{T}_{\Gamma}^{*}=\left\{N_{1}, \ldots, N_{m}\right\}$, set $L_{i}=N_{i}$, for $i=1, \ldots, m$.

If $\gamma$ is a subgraph of $\mu$ and $\mu$ a subgraph of $\Gamma$,

$$
S_{\mu}\left(S_{\gamma} \hat{f}\left(k^{\tau}, q^{\tau} ; a\right)\right)=\hat{f}\left(k^{\tau}\left(k^{\mu}\right), q^{\tau}\left(k^{\mu}, q^{\mu}\right) ; a\right),
$$

or in shorthand notation $S_{\mu} \cdot S_{\gamma}=S_{\mu}$.

For a 1PI subdiagram $\gamma$ of $\Gamma$ we define a Feynman integrand $\hat{I}_{\gamma}$ by

$$
\hat{I}_{\gamma}\left(k^{\gamma}, q^{\gamma} ; \mu, a\right)=\prod_{B \in \mathscr{B}_{\gamma}} \hat{V}_{B}\left(\left\{l_{L}^{\gamma}\right\}_{B} ; \mu, a\right) \cdot \prod_{L \in \mathscr{L}_{\gamma}} \hat{\Delta}_{L}\left(l_{L}^{\gamma} ; \mu, a\right) .
$$

$\left\{l_{L}^{\gamma}\right\}_{B}$ represents the momenta $q_{E}, E \in \mathscr{E}_{\gamma}$ and $\pm l_{L}^{\gamma}, L \in \mathscr{L}_{\gamma}$ flowing into the vertex $B$. We always have

$$
S_{\Gamma} \hat{V}_{B}\left(\left\{l_{L}^{\gamma}\right\}_{B} ; \mu, a\right)=\hat{V}_{B}\left(\left\{l_{L}\right\}_{B} ; \mu, a\right),
$$

and the line momenta are given by (2-16).

Let $\gamma$ be a 1PI subdiagram and $\gamma_{1}, \ldots, \gamma_{c}$ mutually disjoint, connected subdiagrams of $\gamma$. We define the Feynman integrand $\hat{I}_{\bar{\gamma}}$ of the reduced graph $\bar{\gamma}=\gamma / \gamma_{1}, \ldots, \gamma_{c}$ by

$$
\widehat{I}_{\bar{\gamma}}\left(k^{\gamma}, q^{\gamma} ; \mu, a\right)=\prod_{B \in \mathscr{B}_{\bar{\gamma}}} \hat{V}_{B}\left(\left\{l_{L}^{\gamma}\right\}_{B} ; \mu, a\right) \cdot \prod_{L \in \mathscr{L}_{\vec{\gamma}}} \hat{\Delta}_{L}\left(l_{L}^{\gamma} ; \mu, a\right),
$$

where for the reduced vertices $\bar{B}_{i}$ one sets

$$
\hat{V}_{\bar{B}_{\iota}}=1 \in \mathscr{C}^{c} \text { for all } i=1, \ldots, c .
$$

The internal and external momenta of $\bar{\gamma}$ are given by (2-16).

2.3. Divergence Degrees. We now proceed to define UV-divergence degrees for Feynman integrals on the lattice. Let $\gamma$ be a 1PI subdiagram of $\Gamma$. Then the UV-divergence degree of $\gamma$ is given by 
where

$$
\omega(\gamma)=\sum_{L \in \mathscr{L}_{\gamma}} \omega\left(\hat{\Delta}_{L}\right)+\sum_{B \in \mathscr{B}_{\gamma}} \omega\left(\hat{V}_{B}\right)+4 m(\gamma)
$$

$$
\begin{aligned}
& \omega\left(\hat{\Delta}_{L}\right)=\overline{\operatorname{degr}}_{\hat{l}_{L}} \hat{\Delta}_{L}\left(l_{L} ; \mu, a\right), \\
& \omega\left(\hat{V}_{B}\right)=\overline{\operatorname{degr}}_{\left\{\hat{l}_{L}\right\}_{B}} \hat{V}_{B}\left(\left\{l_{L}\right\}_{B} ; \mu, a\right),
\end{aligned}
$$

and $m(\gamma)$ is the number of loops in $\gamma$. The lattice degrees $\overline{\operatorname{degr}}_{\widehat{u}}$ are defined in [3] (see Appendix A) and must be distinguished from the usual UV-degrees $\overline{\operatorname{degr}}_{u}$ of [1], which are defined for rational functions only. In (2-30), they are determined with respect to all momenta entering the vertex $B$. If the functions do not vanish in the limit $a \rightarrow 0$, the UV-degrees on the lattice coincide with the corresponding degrees, defined for rational functions, of the $a \rightarrow 0$-limits of the functions. If the continuum limit of $\hat{V}_{B}$ vanishes and $\hat{V}_{B} \in \mathscr{C}_{m_{0}}$, then $\omega\left(\hat{V}_{B}\right)=m_{0}$ [3].

Similarly, for a reduced diagram $\bar{\gamma}=\gamma / \gamma_{1} \cdots \gamma_{c}$, we set

$$
\omega(\bar{\gamma})=\sum_{L \in \mathscr{L}_{\bar{\gamma}}} \omega\left(\hat{\Delta}_{L}\right)+\sum_{B \in \mathscr{S}_{\bar{\gamma}}} \omega\left(\hat{V}_{B}\right)+4 m(\bar{\gamma})
$$

where $m(\bar{\gamma})$ is the number of loops in $\bar{\gamma}$, and $\omega\left(\hat{\Delta}_{L}\right), \omega\left(\hat{V}_{B}\right)$ are defined as above. For a reduced vertex $\bar{B} \in \mathscr{B}_{\bar{\gamma}}$ we have $\omega\left(\hat{V}_{\bar{B}}\right)=0$ because of $\hat{V}_{\bar{B}}=1$. Furthermore

$$
\begin{aligned}
& m(\bar{\gamma})+\sum_{i=1}^{c} m\left(\gamma_{i}\right)=m(\gamma), \\
& \omega(\bar{\gamma})+\sum_{i=1}^{c} \omega\left(\gamma_{i}\right)=\omega(\gamma) .
\end{aligned}
$$

Finally, we repeat the definition of a forest [1]. Let $\Gamma$ be a 1PI diagram. A $\Gamma$-forest $U$ is a set of 1PI subdiagrams of $\Gamma$ which do not overlap, i.e., for any $\gamma_{1}, \gamma_{2} \in U$ either $\gamma_{1}$ is a subdiagram of $\gamma_{2}$ or $\gamma_{2}$ is a subdiagram of $\gamma_{1}$ or $\gamma_{1}$ and $\gamma_{2}$ are disjoint. In the last case, $\gamma_{1}$ and $\gamma_{2}$ have neither lines nor vertices in common. The simplest forest is given by $U=\emptyset$.

Let $U$ be a $\Gamma$-forest and $\gamma \in U . \gamma$ is called maximal in $U$, if there is no $\gamma^{\prime} \in U$ such that $\gamma$ is a subgraph of $\gamma^{\prime} \cdot \gamma$ is called minimal in $U$, if there exists no $\gamma^{\prime} \in U$ such that $\gamma^{\prime}$ is a subdiagram of $\gamma$. Maximal elements of a forest are disjoint and minimal elements of a forest are also disjoint.

For any 1PI subdiagram $\gamma$ of $\Gamma$ the set of 1PI subdiagrams

$$
U(\gamma)=\left\{\gamma^{\prime} \in U \mid \gamma^{\prime} \text { is a subdiagram of } \gamma \text { and } \gamma^{\prime} \neq \gamma\right\}
$$

is a $\Gamma$-forest as well as a $\gamma$-forest. We define

$$
\bar{\gamma}(U)=\gamma / \gamma_{1} \cdots \gamma_{c}
$$

where $\gamma_{1}, \ldots, \gamma_{c}$ are the maximal elements of $U(\gamma)$.

\section{Subtraction Operators}

Renormalization of Feynman integrals in momentum space can be described by a well-defined procedure of subtractions applied to Feynman integrands. We now define the structure of such subtractions and state their most important properties. 
In the following, let $F$ be a function of the momentum variables $\left(t_{1}, \ldots, t_{h}\right)$, $\left(v_{1}, \ldots, v_{l}\right),\left(q_{1}, \ldots, q_{s}\right)$ and of the lattice spacing $a .(t)$ are the parameters of an affine subspace of the loop momenta, $(v)$ are the complementary loop momenta, and $(q)$ represents the external momenta.

Definition 3.1. Let $\delta \in \mathbf{N}_{0}=\{0,1,2, \ldots\}$ and let $\hat{t}_{q}^{\delta}$ be defined by

$$
\left(\hat{t}_{q}^{\delta} F\right)(t, v, q ; a)=\sum_{n=0}^{\delta} \frac{1}{n !} \sum_{i_{1}, \ldots, i_{n}=0}^{s} P_{n, i_{1} \cdots i_{n}}\left(q_{1}, \ldots, q_{s} ; a\right)\left(\frac{\partial}{\partial q_{i_{1}}} \cdots \frac{\partial}{\partial q_{i_{n}}} F(t, v, q ; a)\right)_{q=0}
$$

for every function $F$ which is $C^{\infty}$ in $q$, where $P_{n, i_{1} \ldots i_{n}} \in \mathscr{C}_{n}^{c}$ are totally symmetric in $i_{1}, \ldots, i_{n},(2 \pi / a)$-periodic in every $q_{1}, \ldots, q_{s}$, and $\lim _{a \rightarrow 0} P_{n, l_{1} \cdots l_{n}}\left(q_{1}, \ldots, q_{s} ; a\right)=$ $q_{i_{1}} \cdots q_{i_{n}}$. If for every such $F$

$$
\left[\left(1-\hat{t}_{q}^{\delta}\right) F\right](t, v, \lambda q ; \mu, a)=O\left(\lambda^{\delta+1}\right) \quad \text { as } \quad \lambda \rightarrow 0,
$$

$\hat{t}_{q}^{\delta}$ is called a subtraction operator of order $\delta$.

If the function $F$ is periodic, then so is $\hat{t}_{q}^{\delta} F$. In the limit $a \rightarrow 0, \hat{t}_{q}^{\delta}$ reproduces the Taylor operator $t_{q}^{\delta}$ of order $\delta$. Note that $\overline{\operatorname{degr}}_{\hat{q}} P_{n, i_{1} \quad i_{n}}(q ; a)=n$ and $\overline{\operatorname{degr}}_{\hat{t}} P_{n, i_{1} \cdots i_{n}}$ $(q ; a)=0 . \hat{t}_{q}^{\delta} F$ can also be written as

$$
\hat{t}_{q}^{\delta} F=\sum_{n=0}^{\delta} \sum_{i_{1}, \ldots, l_{s} \in \mathbf{N}_{0}}^{\prime} \frac{1}{i_{1} ! \cdots i_{s} !} P_{i_{1} \cdots i_{s}}^{(n)}\left(q_{1}, \ldots, q_{s} ; a\right)\left(\frac{\partial^{i_{1}}}{\partial q_{1}^{i_{1}}} \cdots \frac{\partial^{i_{s}}}{\partial q_{s}^{i_{s}}} F\right)_{q=0},
$$

where $P_{i_{1} \cdots i_{s}}^{(n)} \in \mathscr{C}_{n}^{c}$ is periodic in $q_{1}, \ldots, q_{s}$ and $\lim _{a \rightarrow 0} P_{i_{1} \cdots i_{s}}^{(n)}\left(q_{1}, \ldots, q_{s} ; a\right)=q_{1}^{i_{1}} \cdots q_{s}^{i_{s}}$. The inner sum in (3-3) is constrained by $\sum_{j=1}^{s} i_{j}=n$.

In the following, let $F \in \mathscr{F}$ be of the form

$$
\begin{gathered}
F(t, v, q ; \mu, a)=\frac{V(t, v, q ; \mu, a)}{C(t, v, q ; \mu, a)}, \\
V \in \mathscr{C}^{c}, \quad C=\prod_{i=1}^{n}\left[e_{i}\left(l_{i} ; a\right)+\mu_{i}^{2}\right], \quad \mu_{i}^{2}>0, \\
l_{i}=\sum_{k=1}^{l} b_{i k} v_{k}+\sum_{j=1}^{h} c_{i j} t_{j}+\sum_{k=1}^{s} d_{i k} q_{k}, \\
\left(b_{i 1}, \ldots, b_{i l}\right) \neq 0 \quad \text { or } \quad\left(c_{i 1}, \ldots, c_{i h}\right) \neq 0 \text { for all } i=1, \ldots, n .
\end{gathered}
$$

A lattice Feynman integrand $\hat{I}_{\gamma}$ belongs to $\mathscr{F}$. We have assumed all propagators to be massive, hence a subtraction operator $\hat{t}_{q^{\gamma}}^{\delta(\gamma)}$ applies to $\hat{I}_{\gamma}$. The condition (3-2) means that by application of a subtraction operator UV-divergencies are in fact subtracted. It restricts the functions $P_{n, i_{1} \cdots l_{n}}$ and their derivatives at $q=0$. As will be shown in Appendix A, Taylor polynomials in "lattice momenta" satisfy the constraints, i.e. they are special examples of subtraction operators. Actually, Definition 3.1 is much more general.

Besides being linear with respect to scalar multiplication and addition in $\mathscr{F}$, a subtraction operator has the following important properties.

Lemma 3.1. Let $\hat{t}_{q}^{\delta}$ be a subtraction operator of order $\delta$ and $F \in \mathscr{F}$ of the form (3-4). 
Then

1. $\overline{\operatorname{degr}}_{\hat{t} \hat{q}} \hat{t}_{q}^{\delta} F \leqq \overline{\operatorname{degr}}_{\hat{t}} \hat{t}_{q}^{\delta} F+\delta$.

2. $\overline{\operatorname{degr}}_{\hat{t}} \hat{t}_{q}^{\delta} F \leqq \overline{\operatorname{degr}_{\hat{t}}} F$.

If for all $i=1, \ldots, n:\left(c_{i 1}, \ldots, c_{i h}\right)=0 \Rightarrow\left(d_{i 1}, \ldots, d_{i s}\right)=0$, then

3. $\overline{\operatorname{degr}}_{t q} \hat{t}_{q}^{\delta} F \leqq \overline{\operatorname{degr}}_{\hat{t q}} F$.

4. $\overline{\operatorname{degr}}_{\hat{t}}\left(1-\hat{t}_{q}^{\delta}\right) F \leqq \overline{\operatorname{degr}}_{\hat{q} \hat{q}} F-(\delta+1)$.

Proof. To get some experience with lattice degrees, we will do the proof in detail. Especially, we remind the reader of the properties of a UV-degree as stated in [3], Lemma 2.2 (see Appendix A). We will also use multi-index notation. Let $F \in \mathscr{F}$ be of the form (3-4). Note that for arbitrary $V(t, v, q ; \mu, a) \in \mathscr{C}^{c}$,

$$
\begin{gathered}
\left.\overline{\operatorname{degr}}_{\hat{t}} V\right|_{q=0} \leqq \overline{\operatorname{degr}}_{\hat{t}} V, \\
\left.\overline{\operatorname{degr}}_{\hat{t}} V\right|_{q=0}=\left.\overline{\operatorname{degr}}_{\hat{t} \hat{q}} V\right|_{q=0} \leqq \overline{\operatorname{degr}}_{\hat{t} \hat{q}} V .
\end{gathered}
$$

1. Write $\hat{t}_{q}^{\delta} F \in \mathscr{F}$ as

$$
\begin{aligned}
\left(\hat{t}_{q}^{\delta} F\right)(t, v, q ; \mu, a) & =\frac{V^{\prime}(t, v, q ; \mu, a)}{C^{\prime}(t, v ; \mu, a)}, \\
V^{\prime}(t, v, q ; \mu, a) & =\sum_{i_{1}, \ldots, i_{s}=0}^{\delta} \frac{1}{i_{1} ! \cdots i_{s} !} P_{l_{1} \cdots l_{s}}\left(q_{1}, \ldots, q_{s} ; a\right) V_{t_{1} \cdots l_{s}}(t, v ; \mu, a),
\end{aligned}
$$

where the sum is constrained by $\sum_{j=1}^{s} i_{j} \leqq \delta$, and $P_{i_{1}} i_{s}(q ; a) \in \mathscr{C}_{n}^{c}$ for $n=\sum_{j=1}^{s} i_{j}$, $\lim _{a \rightarrow 0} P_{t_{1} i_{s}}(q ; a)=q_{1}^{i_{1}} \cdots q_{s}^{i_{s}}$, and $V_{i_{1} i_{s}} \in \mathscr{C}^{c}$. Because of $\overline{\operatorname{degr}}_{\hat{q} \hat{q}} C^{\prime}=\overline{\operatorname{degr}}_{\hat{f}} C^{\prime}$, it is sufficient to show

$$
\overline{\operatorname{degr}}_{\hat{f} \hat{q}} V^{\prime} \leqq \overline{\operatorname{degr}}_{\hat{f}} V^{\prime}+\delta
$$

In fact,

$$
\begin{aligned}
& \overline{\operatorname{degr}}_{\hat{t} \hat{q}} V^{\prime}(t, v, q ; \mu, a)=\max \overline{\operatorname{degr}}_{\hat{t q}}\left[P_{i_{1} \cdots i_{s}}(q ; a) \cdot V_{\iota_{1} \quad \iota_{s}}(t, v ; \mu, a)\right] \\
& \leqq \delta+\max _{l_{1} \cdot i_{s}} \overline{\operatorname{degr}}{ }_{\hat{t}}\left[P_{t_{1} \cdots t_{s}}(q ; a) \cdot V_{l_{1} \cdots i_{s}}(t, v ; \mu, a)\right] \\
& =\delta+\overline{\operatorname{degr}}_{\hat{t}} V^{\prime}(t, v, q ; \mu, a) .
\end{aligned}
$$

2. Set $l=\left(l_{1}, \ldots, l_{s}\right) \in \mathbf{N}_{0}^{s}$. Then

$$
\overline{\operatorname{degr}}_{\hat{t}}\left[\frac{\partial^{l}}{\partial q^{l}} F(t, v, q ; \mu, a)\right]_{q=0} \leqq \overline{\operatorname{degr}}_{\hat{t}} \frac{\partial^{l}}{\partial q^{l}} F(t, v, q ; \mu, a) \leqq \overline{\operatorname{degr}}_{\hat{t}} F(t, v, q ; \mu, a),
$$

hence

$$
\overline{\operatorname{degr}}_{\hat{t}} P_{n, l_{1} \cdot l_{n}}(q ; a)\left(\frac{\partial^{l}}{\partial q^{l}} F(t, v, q ; \mu, a)\right)_{q=0} \leqq \overline{\operatorname{degr}}_{\hat{t}} F(t, v, q ; \mu, a) .
$$

3. All propagators which depend on $q$ are also dependent on $t$. Hence 


$$
\overline{\operatorname{degr}}_{\hat{q} \hat{q}}\left[\frac{\partial^{l}}{\partial q^{l}} F(t, v, q ; \mu, a)\right]_{q=0} \leqq \overline{\operatorname{degr}}_{\hat{q} \hat{q}} \frac{\partial^{l}}{\partial q^{l}} F(t, v, q ; \mu, a) \leqq \overline{\operatorname{degr}}_{\hat{t} q} F-|l| .
$$

Consequently, for $|l|=n$

$$
\overline{\operatorname{degr}}_{\hat{t} \hat{q}} P_{n, l_{1} \cdots l_{n}}(q ; a)\left(\frac{\partial^{l}}{\partial q^{l}} F(t, v, q ; \mu, a)\right)_{q=0} \leqq n+\overline{\operatorname{degr}}_{\hat{t} \hat{q}} F-|l|=\overline{\operatorname{degr}}_{\hat{t q}} F .
$$

4. $\left(1-\hat{t}_{q}^{\delta}\right) F$ can be written as

$$
\left[\left(1-\hat{t}_{q}^{\delta}\right) F\right](t, v, q ; \mu, a)=\frac{V_{\delta}(t, v, q ; \mu, a)}{C(t, v, q ; \mu, a) C(t, v, 0 ; \mu, a)^{\delta+1}},
$$

where $V_{\delta} \in \mathscr{C}^{c}$. Using (3-3) and $V_{\delta} \in C^{\infty}$, we get $V_{\delta}(t, v, \lambda q ; \mu, a)=O\left(\lambda^{\delta+1}\right)$ as $\lambda \rightarrow 0$, hence

$$
\overline{\operatorname{degr}}_{\hat{t}} V_{\delta} \leqq \overline{\operatorname{degr}}_{\hat{t} \hat{q}} V_{\delta}-(\delta+1) .
$$

By assumption,

$$
\overline{\operatorname{degr}}_{\hat{t}} C(t, v, q ; \mu, a)=\overline{\operatorname{degr}}_{\widehat{t q}} C(t, v, q ; \mu, a) .
$$

Consequently,

$$
\begin{aligned}
\overline{\operatorname{degr}}_{\hat{t}}\left(1-\widehat{t}_{q}^{\delta}\right) F & =\overline{\operatorname{degr}}_{\hat{t}} V_{\delta}(t, v, q ; \mu, a)-\overline{\operatorname{degr}}_{\hat{t}}\left(C(t, v, q ; \mu, a) \cdot C(t, v, 0 ; \mu, a)^{\delta+1}\right) \\
& \leqq \overline{\operatorname{degr}}_{\hat{t} q} V_{\delta}(t, v, q ; \mu, a)-(\delta+1)-\overline{\operatorname{degr}}_{\hat{t} q}\left(C(t, v, q ; \mu, a) \cdot C(t, v, 0 ; \mu, a)^{\delta+1}\right) \\
& =\overline{\operatorname{degr}}_{\hat{q}}\left(1-\hat{t}_{q}^{\delta}\right) F-(\delta+1) \\
& \leqq \overline{\operatorname{degr}}_{\hat{q} q} F-(\delta+1),
\end{aligned}
$$

where we have used Lemma 3.1.3.

\section{Renormalization}

We now proceed to give a renormalization prescription for lattice Feynman integrals with massive propagators. Let

$$
\Gamma=\left(\mathscr{L}_{\Gamma}, \mathscr{E}_{\Gamma}, \mathscr{B}_{\Gamma}, \phi_{\Gamma}, \psi_{\Gamma}\right)
$$

be a 1 PI diagram having $m$ loops, and $\hat{\mathscr{J}}_{\Gamma}(q ; \mu, a)$ the corresponding Feynman integral (2-9). The renormalized Feynman integral of $\Gamma$ is defined by

where

$$
\widehat{\mathscr{R}}_{\Gamma}(q ; \mu, a)=\int_{-\pi / a}^{\pi / a} d^{4} k_{1} \cdots d^{4} k_{m} \hat{R}_{\Gamma}(k, q ; \mu, a),
$$

$$
\hat{R}_{\Gamma}(k, q ; \mu, a)=S_{\Gamma} \sum_{U \in \mathscr{W}} \prod_{\gamma \in U}\left(-\hat{t}_{\gamma}^{\delta(\gamma)} S_{\gamma}\right) \cdot \hat{I}_{\Gamma}(U)
$$

is given as follows:

a. $S_{\gamma}$ are the substitution operators defined in (2-20), (2-22).

b. $\mathscr{W}$ is the set of all $\Gamma$-forests. 
c. $\hat{I}_{\Gamma}(U)$ is the unsubtracted Feynman integrand

$$
\widehat{I}_{\Gamma}(k, q ; \mu, a)
$$

with the following substitutions depending on a forest $U$ : For every line $L \in \mathscr{L}_{\Gamma}$ (vertex $\left.B \in \mathscr{B}_{\Gamma}\right)$ there is at most one $\gamma \in U$ such that $L \in \mathscr{L}_{\gamma}\left(B \in \mathscr{B}_{\gamma}\right)$, but $L \notin \mathscr{L}_{\gamma^{\prime}}$ $\left(B \notin \mathscr{B}_{\gamma^{\prime}}\right)$ for all $\gamma^{\prime} \in U(\gamma)$. If such a $\gamma \in U$ exists, $\hat{\Delta}_{L}\left(\hat{V}_{B}\right)$ is written in variables $q^{\gamma}, k^{\gamma}$ as in (2-16). Otherwise, $\hat{\Delta}_{L}\left(\hat{V}_{B}\right)$ is written as a function of $k, q$ as in (2-8).

d. For every $\gamma, \hat{t}_{\gamma}^{\delta(\gamma)} \equiv \hat{t}_{q^{\gamma}}^{\delta(\gamma)}$ is a subtraction operator of order $\delta(\gamma)$ in external momenta $q^{\gamma}$ of $\gamma . \delta(\gamma)$ is constrained by

$$
\delta(\gamma) \geqq \omega(\gamma),
$$

where $\omega(\gamma)$ is the UV-divergence degree of $\gamma$, and for every $\Gamma$-forest $U$

$$
\delta(\gamma) \geqq \omega(\bar{\gamma}(U))+\sum_{i=1}^{c} \delta\left(\gamma_{i}\right)
$$

where $\gamma_{1}, \ldots, \gamma_{c}$ are the maximal elements of $U(\gamma)$. These conditions are automatically satisfied if $\delta(\gamma)=\omega(\gamma)$ for all $\gamma \in U$. If $\delta(\gamma)<0$, we set $\hat{t}_{\gamma}^{\delta(\gamma)}=0$.

e. In the product

$$
\prod_{\gamma \in U}\left(-\hat{t}_{\gamma}^{\delta(\gamma)} S_{\gamma}\right)
$$

the factors are ordered as follows: If $\gamma_{1}, \gamma_{2} \in U, \gamma_{1}$ subdiagram of $\gamma_{2}$, then $\left(-\hat{t}_{\gamma_{1}}^{\delta\left(\gamma_{1}\right)} S_{\gamma_{1}}\right)$ is ordered to the right-hand side of $\left(-\hat{t}_{\gamma_{2}}^{\delta\left(\gamma_{2}\right)} S_{\gamma_{2}}\right)$. If $\gamma_{1}, \gamma_{2}$ are disjoint, the order is irrelevant.

More explicitly, $\hat{R}_{\Gamma}$ can be written as

$$
\hat{R}_{\Gamma}(k, q ; \mu, a)=\sum_{U \in \mathscr{W}} \hat{R}_{\Gamma}^{U}(k, q ; \mu, a),
$$

where $\hat{R}_{\Gamma}^{U}$ is defined through the following recursion. For minimal $\gamma \in U$, set

$$
\hat{\bar{R}}_{\gamma}^{U}\left(k^{\gamma}, q^{\gamma} ; \mu, a\right)=\hat{I}_{\gamma}\left(k^{\gamma}, q^{\gamma} ; \mu, a\right),
$$

and for every $\gamma \in U \cup\{\Gamma\}, \gamma_{1}, \ldots, \gamma_{c}$ being the maximal elements of $U(\gamma)$,

$$
\hat{\bar{R}}_{\gamma}^{U}\left(k^{\gamma}, q^{\gamma} ; \mu, a\right)=\hat{I}_{\bar{\gamma}(U)}\left(k^{\gamma}, q^{\gamma} ; \mu, a\right) \cdot S_{\gamma} \prod_{i=1}^{c}\left(-\hat{t}_{\gamma_{i}}^{\delta\left(\gamma_{i}\right)}\right) \hat{\bar{R}}_{\gamma_{i}}^{U}\left(k^{\gamma_{1}}, q^{\gamma_{i}} ; \mu, a\right) .
$$

Then $\hat{R}_{\Gamma}^{U}$ is given by

$$
\begin{array}{lll}
\hat{R}_{\Gamma}^{U}=\hat{\bar{R}}_{\Gamma}^{U} & \text { if } & \Gamma \notin U, \\
\hat{R}_{\Gamma}^{U}=-\hat{t}_{\Gamma}^{\delta(\Gamma)} \hat{\bar{R}}_{\Gamma}^{U} & \text { if } & \Gamma \in U .
\end{array}
$$

We now state our main result.

Renormalization Theorem. The continuum limit

$$
\lim _{a \rightarrow 0} \hat{\mathscr{R}}_{\Gamma}(q ; \mu, a)
$$

of the renormalized Feynman integral $\hat{\mathscr{R}}_{\Gamma}(q ; \mu, a)$ exists and is given by

$$
\lim _{a \rightarrow 0} \widehat{\mathscr{R}}_{\Gamma}(q ; \mu, a)=\int_{-\infty}^{\infty} d^{4} k_{1} \cdots d^{4} k_{m} R_{\Gamma}(k, q, \mu),
$$


where

$$
R_{\Gamma}(k, q, \mu)=\lim _{a \rightarrow 0} \hat{R}_{\Gamma}(k, q ; \mu, a) .
$$

Remember that we have assumed all the propagators to be massive. The theorem states that if $\lim _{a \rightarrow 0} \hat{I}_{\Gamma}(k, q ; \mu, a) \neq \equiv, R_{\Gamma}$ is equal to the BPHZ-renormalized continuun limit of $\hat{I}_{\Gamma}$ [1] (with a different choice of internal momenta). If $\lim _{a \rightarrow 0} \hat{I}_{\Gamma}(k, q ; \mu, a) \equiv 0$, also $R_{\Gamma}(k, q, \mu) \equiv 0$. This means that lattice Feynman integrals, which have at least one vertex function with vanishing $a \rightarrow 0$-limit, do not contribute to the continuum limit after renormalization. These vertex functions result from contributions to the lattice action which vanish in the (naive) $a \rightarrow 0$-limit. Such terms do not contribute to the continuum limit in every order of perturbation theory. In this sense, renormalized perturbation theory is universal.

The theorem states that the combinatorics of renormalization of diagrams with a lattice cutoff are given by Zimmermann's forest formula [1], with Taylor subtractions replaced by subtraction operators. The theorem becomes wrong if we would use Taylor operators, since the periodicity of the Feynman integrand, an important convergence condition [3], would be violated. The continuum limit of a renormalized diagram exists and is given by the universal limit resulting from the $a \rightarrow 0$-limit in the integrand. However, it could happen that oversubtractions are necessary, and the higher the loop order, the higher the subtraction degrees. To state conditions which exclude this possibility, we shall write divergence degrees in dependence on terms in the lattice action. Consider an action of the form

$$
\begin{gathered}
S(A)=S_{0}(A)+S_{\mathrm{int}}(A), \\
S_{0}(A)=a^{4} \sum_{n \in \mathbf{Z}^{4}} A_{i}(n a) \hat{\Delta}_{i j}^{-1}(n, a) A_{j}(n a), \\
S_{\mathrm{int}}(A)=a^{4} \sum_{n \in \mathbf{Z}^{4}} \sum_{j} g_{j} L_{j}(A, n a),
\end{gathered}
$$

where $L_{j}$ are polynomials in the lattice spacing $a$, the basic fields $A$ at $n a$ and neighboring lattice sites, and they are homogeneous in $A$. Let

$$
\gamma=\left(\mathscr{L}_{\gamma}, \mathscr{E}_{\gamma}, \mathscr{B}_{\gamma}, \phi_{\gamma}, \psi_{\gamma}\right)
$$

be an arbitrary connected Feynman diagram of a field theory described by $S$. To every line $L \in \mathscr{L}_{\gamma}$ of $\gamma$ there corresponds a pair of basic fields $A_{i}, A_{k}$. We call $L$ an $i k$-type line, having $i$-type and $k$-type legs. For every $A_{i}$ we define a UV-dimension $d_{i}$ such that

$$
4+\omega\left(\hat{\Delta}_{L}\right) \leqq d_{i}+d_{k}
$$

(in four dimensions), i.e. for $i=k$

$$
d_{i} \geqq \frac{1}{2}\left[\omega\left(\hat{\Delta}_{L}\right)+4\right] .
$$

The number of loops in $\gamma$ is given by

Hence

$$
m(\gamma)=1+\sum_{L \in \mathscr{L}_{\gamma}} 1-\sum_{B \in \mathscr{B}_{\gamma}} 1 .
$$

$$
\omega(\gamma)=4+\sum_{L \in \mathscr{L}_{\gamma}}\left(\omega\left(\hat{\Delta}_{L}\right)+4\right)+\sum_{B \in \mathscr{B}_{\gamma}}\left(\omega\left(\hat{V}_{B}\right)-4\right) .
$$


After some elementary manipulations, using (4-11), we get (cp. [6])

where

$$
\begin{aligned}
& \omega(\gamma) \leqq \bar{\omega}(\gamma), \\
& \bar{\omega}(\gamma)=4+\sum_{B \in B_{\gamma}}(\omega(B)-4)-\sum_{k} e_{k}(\gamma) d_{k},
\end{aligned}
$$

$$
\omega(B)=\sum_{k} n_{k}(B) d_{k}+\omega\left(\hat{V}_{B}\right) .
$$

$n_{k}(B)$ is the number of $k$-type legs entering the vertex $B$ or synonymously is the power of $k$-type fields $A_{k}$ in the action term corresponding to the vertex $B . \omega(B)$ can be determined directly from this part of the action. $e_{k}(\gamma)$ is the number of external $k$-type lines of $\gamma$.

From these expressions, it is directly seen that the constraints (4-3) and (4-4) are satisfied if we choose

$$
\delta(\gamma)=4+\sum_{B \in \mathscr{B}_{\gamma}}(\delta(B)-4)-\sum_{k} e_{k}(\gamma) d_{k} \quad \text { with } \quad \delta(B) \geqq \omega(B) .
$$

Consequently, a field theory on the lattice is renormalizable by power counting if for every vertex $B$ the UV-divergence degree of $B$ satisfies $\omega(B) \leqq 4$. (Renormalizable by power counting means that with increasing number of loops the order of subtractions needed does not increase.) In particular, we can state the following

Theorem. Let the coupling constants $g_{j}$ in (4-10) be dimensionless. Take the limit $a \rightarrow 0$ of (4-8) and denote the resulting continuum action by $S_{c}(A)$. If for every vertex $B$ of $S_{c}(A)$ the continuum UV-divergence degree $\omega_{c}(B)[6]$ satisfies $\omega_{c}(B) \leqq 4$, then the lattice theory is renormalizable, and its continuum limit is given by the field theory which is described by the action $S_{c}(A)$, and is renormalized by the BPHZ finite part prescription.

As an example, consider the scalar $\Phi^{4}$-theory with an additional $\Phi^{6}$-interaction:

$$
S(\Phi)=a^{4} \sum_{n \in \mathbf{Z}^{4}}\left[\Phi(n a)\left(-\hat{\square}+\mu^{2}\right) \Phi(n a)+g \Phi^{4}(n a)+\lambda a^{2} \Phi^{6}(n a)\right] .
$$

The propagator in this example is given by

where

$$
\hat{\Delta}(k)=\frac{1}{\hat{k}^{2}+\mu^{2}},
$$

$$
\hat{k}_{i}=\frac{2}{a} \sin \frac{k_{i} a}{2}, \quad i=1, \ldots, 4, \quad \hat{k}^{2}=\sum_{i=1}^{4}\left(\hat{k}_{i}\right)^{2} .
$$

The only Green functions to be renormalized are the two- and four-point functions. In fact, any vertex $B$ satisfies $\omega(B)=4$, and (4-14) shows that the divergence degrees of six-point and higher functions are smaller than zero. The four-point Green function has divergence degree $\omega=0$. Hence subtraction of a constant is sufficient to absorb its overall divergence. The two-point function is quadratically divergent, and to renormalize it, we should choose as a subtraction operator $\hat{t}_{q}^{2}$ a Taylor operator of order two in the lattice momenta $\hat{q}$. If $\hat{q}$ would be periodic, this is an always allowed choice (as shown in Appendix A). However, $\hat{q}$ is anti-periodic with the $\mathrm{BZ}$, barring at the first sight $\hat{t}_{q}^{2}$ to be a proper subtraction operator. Actually, 
the model (4-17) is invariant under inversion $q \rightarrow-q$. This means, the first and mixed second derivatives to external momenta of diagrams vanish at zero momenta. The "effective" subtraction operator is given by

$$
\hat{t}_{q}^{2}=1+\frac{1}{2} \sum_{i=1}^{s} \hat{q}_{i}^{2}\left[\frac{\partial^{2}}{\partial \hat{q}_{i}^{2}}\right]_{q=0},
$$

which is periodic. The renormalization theorem states that the $a \rightarrow 0$-limit of the renormalized model of (4-17) exists and is described by the action

$$
S_{c}(\Phi)=\int d^{4} x\left[\Phi(x)\left(-\square+\mu^{2}\right) \Phi(x)+g \Phi^{4}(x)\right],
$$

and renormalized by the BPHZ finite part prescription. The limit is independent of the coupling $\lambda$.

If at any order the overall subtractions of diagrams could be written as counterterms in the lattice action, then they subtract in higher orders divergencies of corresponding subdiagrams. However, for a lattice cutoff, the situation is a bit more involved. To apply $\hat{t}_{q^{\gamma}}^{\delta(\gamma)}$ to a diagram $\gamma$, we have to choose a basis of the external momenta of $\gamma$. By momentum conservation, one line is omitted, but which line is arbitrary. A similar arbitrariness holds for the coefficient functions $P_{n, l_{1} l_{n}}$ in $\hat{t}_{q^{\prime}}^{\delta(\gamma)}$. The differences are always of order $O(a)$, and by the renormalization theorem, they do not have any influence on the continuum limit. Note that in the continuum the problem does not occur, subtractions being Taylor polynomials and hence independent of a basis.

To get a counterterm in the action, we have to respect Bose and Fermi symmetries under exchange of equal-type external lines. This can be achieved as follows. First of all, we have to choose the same subtraction operator for all diagrams $\gamma$, which differ by an exchange of equal-type external lines only. This subtraction operator must be chosen to be symmetric, i.e. if it is written in a form (3-3), then for any permutation $\pi$ of equal-type lines

$$
P_{i_{\pi(1)} \cdot i_{\pi(N)}}^{(n)}\left(q_{\pi(1)}, \ldots, q_{\pi(N)} ; a\right)=P_{i_{1} \cdot{ }_{1}}^{(n)}\left(q_{1}, \ldots, q_{N} ; a\right),
$$

for all $i_{1}, \ldots, i_{N}$, where $N+1$ is the number of external lines of $\gamma$. Finally, we have to take the arithmetic mean over all possible bases, or at least over those bases omitting an external line of the same type. Then the counterterm which results by integration over all loop momenta has the same permutation symmetries as the unsubtracted $\gamma$. Summation over all diagrams, which differ by an exchange of equal-type external lines, yields a counterterm having the desired Bose or Fermi symmetries, and it can be written as a contribution to the action.

Furthermore, if the functions

$$
P_{t_{1} t_{N}}^{(n)}\left(q_{1}, \ldots, q_{N} ; a\right)
$$

are chosen to be symmetric and polynomials in lattice momenta (see Appendix A), the counterterms are always local, i.e., they are of the form

$$
\Delta \Gamma_{\text {eff }}=a^{4} \sum_{n \in \mathbf{Z}^{4}} P(A, n a),
$$

where $P(A, n a)$ is a homogeneous polynomial in the fields $A$ at $n a$ and neighboring lattice sites. 


\section{Convergence Proof}

To prove the renormalization theorem, we will show that (4-1) satisfies all criteria of the power counting theorem of [3]. The subtracted integrand (4-2) can be written as

where

$$
\hat{R}_{\Gamma}(k, q ; \mu, a)=\frac{V(k, q ; \mu, a)}{B_{1}(k, q ; \mu, a) B_{2}(k ; \mu, a)},
$$

$$
\begin{aligned}
B_{1}(k, q ; \mu, a) & =\prod_{L \in \mathscr{L}_{\Gamma}} \prod_{j=1}^{n(L)}\left(e_{L j}\left(l_{L} a\right)+\mu_{L j}^{2}\right), \\
B_{2}(k ; \mu, a) & =\prod_{\gamma} \prod_{L \in \mathscr{L}_{\gamma}} \prod_{j=1}^{n(L)}\left(e_{L j}\left(k_{L}^{\gamma} a\right)+\mu_{L j}^{2}\right)^{n_{j}(L, \gamma)},
\end{aligned}
$$

all masses $\mu_{L j}$ are nonvanishing, $n_{j}(L, \gamma) \in \mathbf{N}_{0}=\{0,1,2, \ldots\}$, and the outer product is over all 1PI subdiagrams $\gamma$ of $\Gamma$. Furthermore,

$$
l_{L}(k, q)=k_{L}(k)+q_{L}(q), \quad k_{L}^{\gamma}=k_{L}^{\gamma}(k),
$$

and $V \in \mathscr{C}^{c}$, i.e., $\hat{R}_{\Gamma}$ belongs to $\mathscr{F}$ (see Appendix A). By definition of the subtraction operators and of $S_{\gamma}, \hat{R}_{\Gamma}$ is periodic in the loop momenta $k_{1}, \ldots, k_{m}$.

Let $\mathscr{L}$ be the set of all $l_{L}, L \in \mathscr{L}_{\Gamma}$, and of all $k_{L}^{\gamma}$ for 1PI subdiagrams $\gamma$ of $\Gamma$ and $L \in \mathscr{L}_{\gamma}$. By construction of $k_{L}^{\gamma}$, the set $\mathscr{L}$ is natural in the sense of [3]. All that remains to be shown is that the power counting conditions of [3] are satisfied. Let

$$
t_{1}, \ldots, t_{h}, \quad v_{1}, \ldots, v_{m-h}
$$

be an arbitrary basis of $\mathscr{L}$, i.e. $t_{1}, \ldots, t_{h}, v_{1}, \ldots, v_{m-h} \in \mathscr{L}$, and the Jacobian satisfies $\operatorname{det}(\partial(t, v) / \partial(k)) \neq 0$. By fixing $v_{1}, \ldots, v_{m-h}$, one defines a Zimmermann subspace $H$, i.e. a class of affine subspaces of the space of loop momenta $\left(k_{1}, \ldots, k_{m}\right) . k=k(t, v, q)$ and $k^{\gamma}=k^{\gamma}(t, v, q)$ for every 1PI subdiagram $\gamma$ of $\Gamma$ are linear functions. $(t)=\left(t_{1}, \ldots, t_{h}\right)$ is called the parametrization of $H$. The set of all such Zimmermann subspaces $H$, for all bases (5-4), is denoted by $\mathscr{H}$. We will show that for every $H \in \mathscr{H}$, parametrized by $(t)=\left(t_{1}, \ldots, t_{h}\right)$ with respect to a basis (5-4) of $\mathscr{L}$

$$
4 h+\overline{\operatorname{degr}}_{\hat{t}} \hat{R}_{\Gamma}(k(t, v, q), q ; \mu, a)<0 .
$$

Then all the conditions are met for the power counting theorem to apply to the renormalized Feynman integral (4-1). This concludes the proof of the renormalization theorem.

The general idea of proof can be found in [1] and uses the method of so-called complete forests. What is different here is the form of the integrand, a new kind of subtraction and the definition of a UV-divergence degree. However, as will be seen below, (5-5) is based on general properties of the divergence degrees [3], and of the subtraction operators (Lemma 3.1). This allows us to use the ideas of [1] (cp. [4]). Especially, the combinatorical part of the proof can be taken over literally. At first, we have to repeat the definition of a complete forest. A $\Gamma$-forest $U \in \mathscr{W}$ is called complete on $H$, parametrized by $(t)$, if $\Gamma \in U$, and if for any $\gamma \in U$ : all lines of $\bar{\gamma}(U)$ are constant on $H$ relative to $\gamma$, i.e.

$$
k_{L}^{\gamma}(t, v, q) \quad \text { is independent of } t \text { for every } \quad L \in \mathscr{L}_{\bar{\gamma}(U)} \text {, or }
$$


all lines of $\bar{\gamma}(U)$ are variable on $H$ relative to $\gamma$, i.e.

$$
k_{L}^{\gamma}(t, v, q) \text { is dependent on } t \text { for every } L \in \mathscr{L}_{\bar{\gamma}(U)} \text {. }
$$

$\bar{\gamma}(U)$ is said to be constant or variable on $H$, respectively. The forest formula (4-2) can now be written in a form dependent on a given Zimmermann subspace $H$.

Lemma 5.1 [1]. Let $\Gamma$ be a 1 PI diagram and $H \in \mathscr{H}$. Then

$$
\hat{R}_{\Gamma}(k, q ; \mu, a)=\sum_{U \in \mathscr{H}_{c}^{H}} \hat{X}_{U}(k, q ; \mu, a)
$$

where $\mathscr{W}_{c}^{H}$ is the set of all $\Gamma$-forests which are complete on $H . \hat{X}_{U}$ is recursively determined by

$$
\hat{X}_{U}(k, q ; \mu, a)=\left.\left(1-\hat{t}_{\Gamma}^{\delta(\Gamma)}\right) \hat{Y}_{\Gamma}\left(k^{\Gamma}, q^{\Gamma} ; \mu, a\right)\right|_{k^{\Gamma}=k, q^{\Gamma}=q},
$$

where for minimal $\gamma \in U$

$$
\hat{Y}_{\gamma}\left(k^{\gamma}, q^{\gamma} ; \mu, a\right)=\hat{I}_{\gamma}\left(k^{\gamma}, q^{\gamma} ; \mu, a\right)
$$

and for any other $\gamma \in U$

$$
\hat{Y}_{\gamma}\left(k^{\gamma}, q^{\gamma} ; \mu, a\right)=\hat{I}_{\overline{\gamma(U)}}\left(k^{\gamma}, q^{\gamma} ; \mu, a\right) \cdot S_{\gamma} \prod_{i=1}^{c} \hat{f}\left(\gamma_{i}\right) \hat{Y}_{\gamma_{t}}\left(k^{\gamma_{1}}, q^{\gamma_{1}} ; \mu, a\right) .
$$

$\gamma_{1}, \ldots, \gamma_{c}$ are the maximal elements of $U(\gamma) . \hat{f}(\gamma)$ is defined by

$$
\hat{f}(\gamma)=\left\{\begin{array}{lll}
1-\hat{t}_{\gamma}^{\delta(\gamma)} & \text { if } & \gamma \in \mathscr{B}(U) \\
-\hat{t}_{\gamma}^{\delta(\gamma)} & \text { if } & \gamma \notin \mathscr{B}(U) .
\end{array}\right.
$$

$\mathscr{B}(U)$ is the set of all $\gamma \in U$, which have $\bar{\gamma}(U)$ variable on $H$ and in addition are maximal element of $U(\tau)$ for some $\tau \in U$ having $\bar{\tau}(U)$ constant on $H$.

All functions $\hat{Y}_{\gamma}, \hat{X}_{U}, \hat{R}_{\Gamma}$ are in $\mathscr{F}$. The $H$-dependent form (5-6) of $\hat{R}_{\Gamma}$ allows an estimation of $\overline{\operatorname{degr}}_{\hat{t}} \hat{R}_{\Gamma}$ by induction through a complete forest. As will be seen, every single term in the sum (5-6) already satisfies the desired bound on the UV-degree. The proof of Lemma 5.1 can be found in [1]. What is different here are the structure of the functions and the definition of internal and external momenta of subdiagrams. However, this does not have any influence onto the validity of Lemma 5.1 which is mainly a combinatorical statement. Of importance is that the internal momenta of subdiagrams are determined always in the same way. This is guaranteed by (2-24).

For every $U \in \mathscr{W}_{c}^{H}$ and every $\gamma \in U$ we set

$$
M_{U}(\gamma)=4 \sum_{\tau} m(\bar{\tau}(U))
$$

where $\tau \in U(\gamma) \cup\{\gamma\}, \bar{\tau}(U)$ variable on $H$, and where $m(\bar{\tau}(U))$ is the number of loops in $\bar{\tau}(U) . M_{U}(\Gamma)$ sums up the number of independent parameters of $H$, i.e., $M_{U}(\Gamma) \geqq 4 h$. This is proved in Appendix C. We now state the important

Lemma. 5.2. Let $H \in \mathscr{H}$ with parametrization $(t)=\left(t_{1}, \ldots, t_{h}\right), U$ a $\Gamma$-forest which is complete on $H$, and $\hat{R}_{\Gamma}, \hat{Y}_{\gamma}$ as in Lemma 5.1. Then for every $\gamma \in U$ :

1. $\overline{\operatorname{degr}}_{\hat{\imath}} \hat{Y}_{\gamma}\left(k^{\gamma}(t, v, q), q^{\gamma} ; \mu, a\right) \leqq-M_{U}(\gamma)$ for $\bar{\gamma}(U)$ constant, equality holding only if $M_{U}(\gamma)=0$. 
2. $\overline{\operatorname{degr}}_{\hat{t}^{\gamma}} \hat{Y}_{\gamma}\left(k^{\gamma}(t, v, q), q^{\gamma} ; \mu, a\right) \leqq \delta(\gamma)-M_{U}(\gamma)$ for $\bar{\gamma}(U)$ variable.

From 1. and 2. we get

$$
\overline{\operatorname{degr}}_{\hat{t}} \hat{R}_{\Gamma}(k(t, v, q), q ; \mu, a) \leqq-4 h-1 .
$$

Note that the dependence of $k^{\gamma}$ on the external momenta $q$ does only occur through the parametrization of a Zimmermann subspace $H$. Differentiations and UV-degrees with respect to $q^{\gamma}$ refer only to the explicit $q^{\gamma}$-dependence. Statement (5-13) is the desired power counting condition. Because $H$ is an arbitrary subspace in $\mathscr{H}$, all conditions of the power counting theorem of [3] are satisfied. Hence, from Lemma 5.2 the renormalization theorem follows.

Proof. By induction through the forest $U$. We will permanently use the degree properties of Lemma 2.2 of [3] without explicit reference. $\hat{t}_{\gamma}^{\delta(\gamma)}$ is a subtraction operator to which Lemma 3.1 applies. Note that the numerator $V(t, v, q ; \mu, a)$ of a function $V / C \in \mathscr{F}$ satisfies $\overline{\operatorname{degr}}_{\widehat{t q}} V \leqq \overline{\operatorname{degr}}_{\widehat{t r q}} V$, and if $V$ is independent of $t$, then $\overline{\operatorname{degr}}_{\hat{t}} V \leqq 0$. If $\gamma$ is minimal, then $\bar{\gamma}(U)=\gamma$ and $\hat{Y}_{\gamma}=\hat{I}_{\gamma}$, hence

$\overline{\operatorname{degr}}_{\hat{t}} \hat{Y}_{\gamma}\left(k^{\gamma}(t, v, q), q^{\gamma} ; \mu, a\right) \leqq 0 \quad$ if $\bar{\gamma}(U)$ is constant,

$\overline{\operatorname{degr}}_{\hat{i q}^{\gamma}} \hat{Y}_{\gamma}\left(k^{\gamma}(t, v, q), q^{\gamma} ; \mu, a\right) \leqq \omega(\gamma)-4 m(\gamma) \leqq \delta(\gamma)-M_{U}(\gamma)$ if $\bar{\gamma}(U)$ is variable.

Now we assume that the inequalities 1. and 2. hold for all maximal elements $\gamma_{i} \in U(\gamma), i=1, \ldots, c$, for some non-minimal $\gamma \in U$. Then $\hat{Y}_{\gamma}$ is given in $(5-8 \mathrm{~b})$. $S_{\gamma}$ means a linear substitution

$$
k^{\gamma_{2}} \rightarrow k^{\gamma_{1}}\left(k^{\gamma}\right), \quad q^{\gamma_{2}} \rightarrow q^{\gamma_{2}}\left(k^{\gamma}, q^{\gamma}\right) .
$$

1. Let $\bar{\gamma}(U)=\gamma / \gamma_{1} \cdots \gamma_{c}$ be constant on $H$. Then

a.

$$
\overline{\operatorname{degr}} \hat{I}_{\gamma / \gamma_{1} \cdots \gamma_{c}}\left(k^{\gamma}(t, v, q), q^{\gamma} ; \mu, a\right) \leqq 0 .
$$

b. $\bar{\gamma}_{i}(U)$ constant. Then $\hat{f}\left(\gamma_{i}\right)=-\hat{t}_{\gamma_{i}}^{\delta\left(\gamma_{i}\right)}$. According to the hypothesis of induction

$$
\overline{\operatorname{degr}} \hat{Y}_{\hat{\imath}} \hat{Y}_{\gamma_{t}}\left(k^{\gamma_{t}}(t, v, q), q^{\gamma_{t}} ; \mu, a\right) \leqq-M_{U}\left(\gamma_{i}\right)
$$

(equality holding only if $M_{U}\left(\gamma_{i}\right)=0$ ). Hence

$$
\begin{aligned}
& \overline{\operatorname{degr}}_{\hat{t}}\left(-\hat{t}_{\gamma_{1}}^{\delta\left(\gamma_{2}\right)}\right) \hat{Y}_{\gamma_{i}}\left(k^{\gamma_{i}}(t, v, q), q^{\gamma_{2}} ; \mu, a\right) \leqq-M_{U}\left(\gamma_{i}\right) \quad \text { by Lemma 3.1.2, } \\
& \overline{\operatorname{degr}_{\hat{t}}} S_{\gamma}\left(-\hat{t}_{\gamma_{2}}^{\delta\left(\gamma_{t}\right)}\right) \hat{Y}_{\gamma_{i}}\left(k^{\gamma_{2}}(t, v, q), q^{\gamma_{i}} ; \mu, a\right) \\
& =\overline{\operatorname{degr}}_{\hat{t}}\left[S_{\gamma}\left(-\hat{t}_{\gamma_{t}}^{\delta\left(\gamma_{t}\right)}\right) \hat{Y}_{\gamma_{t}}\right]\left(k^{\gamma}(t, v, q), q^{\gamma} ; \mu, a\right) \leqq-M_{U}\left(\gamma_{i}\right) \\
& \quad\left(=\text { holding only if } M_{U}\left(\gamma_{i}\right)=0\right) .
\end{aligned}
$$

For, $q^{\gamma_{1}}$ depends via $S_{\gamma}$ only on those $k^{\gamma}$ which are constant on $H$ relative to $\gamma$, i.e., $q^{\gamma_{2}}$ is independent of $t$.

c. $\bar{\gamma}_{i}(U)$ variable. Then $\hat{f}\left(\gamma_{i}\right)=1-\hat{t}_{\gamma_{t}}^{\delta\left(\gamma_{2}\right)}$, and according to the hypothesis of induction

$$
\overline{\operatorname{degr}}_{t q}{\widehat{\gamma_{i}}}_{\hat{Y}_{\gamma_{t}}}\left(k^{\gamma_{t}}(t, v, q), q^{\gamma_{1}} ; \mu, a\right) \leqq \delta\left(\gamma_{i}\right)-M_{U}\left(\gamma_{i}\right)
$$

$\hat{Y}_{\gamma_{t}}$ is of the form 


$$
\hat{Y}_{\gamma_{i}}\left(k^{\gamma}, q^{\gamma} ; \mu, a\right)=\hat{I}_{\gamma_{i} / \gamma_{i 1} \cdot \gamma_{i d}}\left(k^{\gamma}, q^{\gamma} ; \mu, a\right) S_{\gamma_{i}} \prod_{j=1}^{d}\left(-\hat{t}_{\gamma_{i j}}^{\delta\left(\gamma_{i j}\right)}\right) \hat{Y}_{\gamma_{i j}}\left(k^{\gamma_{i j}}, q^{\gamma_{\gamma_{j}}} ; \mu, a\right) .
$$

All lines of $\bar{\gamma}_{i}(U)=\gamma_{i} / \gamma_{i 1} \cdots \gamma_{i d}$ are variable on $H$ relative to $\gamma_{i}$, and the denominator of

$$
S_{\gamma_{2}} \prod_{j=1}^{d}\left(-\hat{t}_{\gamma_{\gamma_{j}}}^{\delta\left(\gamma_{\gamma_{j}}\right)}\right) \hat{Y}_{\gamma_{i j}}\left(k^{\gamma_{\gamma_{j}}}, q^{\gamma_{i_{j}}} ; \mu, a\right)
$$

does not depend on $q^{\gamma_{1}}, k^{\gamma_{l_{j}}}=k^{\gamma_{t_{J}}}\left(k^{\gamma_{1}}\right)$ being independent of $q^{\gamma_{1}}$ via $S_{\gamma_{1}}$. Hence Lemma 3.1.4 applies to $\hat{Y}_{\gamma_{i}}$ :

$$
\overline{\operatorname{degr}}_{\hat{t}}\left(1-\hat{t}_{\gamma_{i}}^{\delta\left(\gamma_{i}\right)}\right) \hat{Y}_{\gamma_{i}}\left(k^{\gamma_{i}}(t, v, q), q^{\gamma_{i}} ; \mu, a\right) \leqq-M_{U}\left(\gamma_{i}\right)-1 .
$$

Again, in $q^{\gamma_{t}}$ only those $k^{\gamma}$ occur which are constant on $H$, hence

$$
\overline{\operatorname{degr}_{\hat{t}}} S_{\gamma}\left(1-\hat{t}_{\gamma_{2}}^{\delta\left(\gamma_{1}\right)}\right) \hat{Y}_{\gamma_{i}}\left(k^{\gamma_{1}}(t, v, q), q^{\gamma_{1}} ; \mu, a\right) \leqq-M_{U}\left(\gamma_{i}\right)-1 .
$$

In summary

$$
\overline{\operatorname{degr}}_{\hat{t}} \hat{Y}_{\gamma}\left(k^{\gamma}(t, v, q), q^{\gamma} ; \mu, a\right) \leqq-M_{U}(\gamma) \quad\left(=\text { holding only if } M_{U}(\gamma)=0\right),
$$

where we have used

$$
M_{U}(\gamma)=\sum_{i=1}^{c} M_{U}\left(\gamma_{i}\right) \quad(\bar{\gamma}(U) \text { constant })
$$

2. Let $\bar{\gamma}(U)$ be variable on $H$. For all $i=1, \ldots, c: \hat{f}\left(\gamma_{i}\right)=-\hat{t}_{\gamma_{t}}^{\delta\left(\gamma_{t}\right)}$.

a.

$$
\overline{\operatorname{degr}} \widehat{A}_{1 q^{\gamma}} \hat{I}_{\gamma_{\gamma_{1}} \cdot \gamma_{c}}\left(k^{\gamma}(t, v, q), q^{\gamma} ; \mu, a\right) \leqq \omega(\bar{\gamma}(U))-4 m(\bar{\gamma}(U))
$$

by definition of $\omega(\bar{\gamma}(U))$ and $m(\bar{\gamma}(U))$.

b. $\bar{\gamma}_{i}(U)$ constant.

$$
\begin{aligned}
& \overline{\operatorname{degr}}_{\hat{t}} \hat{Y}_{\gamma_{i}}\left(k^{\gamma_{2}}(t, v, q), q^{\gamma_{2}} ; \mu, a\right) \leqq-M_{U}\left(\gamma_{i}\right) \quad\left(=\text { only if } M_{U}\left(\gamma_{i}\right)=0\right), \\
& \overline{\operatorname{degr}}_{\hat{i}}\left(-\hat{t}_{\gamma_{i}}^{\delta\left(\gamma_{i}\right)}\right) \hat{Y}_{\gamma_{i}}\left(k^{\gamma_{i}}(t, v, q), q^{\gamma_{i}} ; \mu, a\right) \leqq-M_{U}\left(\gamma_{i}\right) \text { by Lemma 3.1.2, } \\
& \overline{\operatorname{degr}} \widehat{\widehat{r}}_{q_{1}}\left(-\widehat{t}_{\gamma_{t}}^{\delta\left(\gamma_{2}\right)}\right) \hat{Y}_{\gamma_{i}}\left(k^{\gamma_{2}}(t, v, q), q^{\gamma_{i}} ; \mu, a\right) \leqq \delta\left(\gamma_{i}\right)-M_{U}\left(\gamma_{i}\right) \text { by Lemma 3.1.1. }
\end{aligned}
$$

The denominator does not depend on $q^{\gamma_{2}}$, and linear $S_{\gamma}$ can only decrease the degree with respect to $\left(t, q^{\gamma}\right)$ :

$$
\overline{\operatorname{degr}} \widehat{\widehat{l i}}_{i \gamma^{\gamma}} S_{\gamma}\left(-\hat{t}_{\gamma_{2}}^{\delta\left(\gamma_{i}\right)}\right) \hat{Y}_{\gamma_{i}}\left(k^{\gamma_{i}}(t, v, q), q^{\gamma_{1}} ; \mu, a\right) \leqq \delta\left(\gamma_{i}\right)-M_{U}\left(\gamma_{i}\right)
$$

c. $\bar{\gamma}_{i}(U)$ variable.

$$
\begin{aligned}
& \overline{\operatorname{degr}}_{1 q^{\gamma_{i}}} \hat{Y}_{\gamma_{t}}\left(k^{\gamma_{i}}(t, v, q), q^{\gamma_{i}} ; \mu, a\right) \leqq \delta\left(\gamma_{i}\right)-M_{U}\left(\gamma_{i}\right) \quad \text { (induction hypothesis), }
\end{aligned}
$$

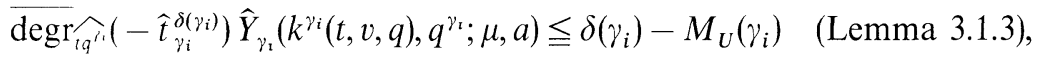

using the same arguments as for 1.c,

$$
\overline{\operatorname{degr}}_{\hat{q q}^{\gamma}} S_{\gamma_{\gamma}}\left(-\hat{t}_{\gamma_{i}}^{\delta\left(\gamma_{i}\right)}\right) \hat{Y}_{\gamma_{i}}\left(k^{\gamma_{1}}(t, v, q), q^{\gamma_{1}} ; \mu, a\right) \leqq \delta\left(\gamma_{i}\right)-M_{U}\left(\gamma_{i}\right) .
$$

In summary

$$
\overline{\operatorname{degr}_{\text {tq }}{ }_{q^{\gamma}}} \hat{Y}_{\gamma}\left(k^{\gamma}(t, v, q), q^{\gamma} ; \mu, a\right) \leqq \omega(\bar{\gamma}(U))+\sum_{i=1}^{c} \delta\left(\gamma_{i}\right)-M_{U}(\gamma)
$$


where we have used $M_{U}(\gamma)=4 m(\bar{\gamma}(U))+\sum_{i=1}^{c} M_{U}\left(\gamma_{i}\right)$. According to the condition (4-4) this implies

$$
\overline{\operatorname{degr}} \widehat{t q}_{q^{\gamma}} \hat{Y}_{\gamma}\left(k^{\gamma}(t, v, q), q^{\gamma} ; \mu, a\right) \leqq \delta(\gamma)-M_{U}(\gamma) .
$$

To prove the last statement of the lemma, we must distinguish between $\bar{\Gamma}(U)$ variable and $\bar{\Gamma}(U)$ constant. If $\bar{\Gamma}(U)$ is constant on $H$, then

$$
\overline{\operatorname{degr}}_{\hat{t}} \hat{Y}_{\Gamma}\left(k^{\Gamma}(t, v, q), q^{\Gamma} ; \mu, a\right) \leqq-M_{U}(\Gamma)-1 \leqq-4 h-1,
$$

hence, using Lemma 3.1.2

$$
\overline{\operatorname{degr}}_{\hat{t}} \hat{X}_{U}(k(t, v, q), q ; \mu, a) \leqq \overline{\operatorname{degr}}_{\hat{t}}\left(1-\hat{t}_{\Gamma}^{\delta(\Gamma)}\right) \hat{Y}_{\Gamma}\left(k^{\Gamma}(t, v, q), q^{\Gamma} ; \mu, a\right) \leqq-4 h-1 .
$$

If $\bar{\Gamma}(U)$ is variable on $H$,

$$
\overline{\operatorname{degr}} \widehat{t q}^{I} \hat{Y}_{\Gamma}\left(k^{\Gamma}(t, v, q), q^{\Gamma} ; \mu, a\right) \leqq \delta(\Gamma)-M_{U}(\Gamma) \leqq \delta(\Gamma)-4 h .
$$

Using Lemma 3.1.4 and the same argument as for 1.c, we get

$$
\overline{\operatorname{degr}}_{\hat{t}} \hat{X}_{U}(k(t, v, q), q ; \mu, a) \leqq \overline{\operatorname{degr}}_{\hat{t}}\left(1-\hat{t}_{\Gamma}^{\delta(\Gamma)}\right) \hat{Y}_{\Gamma}\left(k^{\Gamma}(t, v, q), q^{\Gamma} ; \mu, a\right) \leqq-4 h-1 .
$$

Hence in both cases

$$
\overline{\operatorname{degr}}_{\hat{t}} \hat{R}_{\Gamma}(k(t, v, q), q ; \mu, a) \leqq-4 h-1,
$$

and Lemma 5.2 is completely proved.

\section{Generalizations}

Until now, we have discussed Feynman integrals for scalar fields only. This we have done for simplicity. There is no essential change if we include fields carrying spin and internal symmetries like Lorentz, colour, spinor indices etc. The diagrammatic notations introduced in Sect. 2 are supplemented by the notion of an index distribution.

Definition 6.1. Let $\Gamma$ be a Feynman diagram as given in (2-1). An index distribution is a collection of two maps $\mathscr{A}_{1}, \mathscr{A}_{2}$, defined by

$$
L \stackrel{\mathscr{A}_{1}}{\longrightarrow} \Lambda_{L}^{1} \times \Lambda_{L}^{2} \text { for all } L \in \mathscr{L}_{\Gamma}, \quad E \stackrel{\mathscr{A}_{2}}{\longrightarrow} \Lambda_{E} \text { for all } E \in \mathscr{E}_{\Gamma} \text {. }
$$

All $\Lambda$ 's are finite sets.

The index sets $\Lambda$ are carrying the symmetry labels. Note that to every internal line there correspond two indices, one for each end of the line. In calculations, these indices are summed over. Propagators and vertex functions are now dependent on momenta and indices. A Feynman integral has the form

$$
\begin{gathered}
\mathscr{J}^{\Gamma}\left(q, \alpha\left(\mathscr{E}_{\Gamma}\right) ; \mu, a\right)=\sum_{\alpha_{L}, \beta_{L}} \int_{-\pi / a}^{\pi / a} d^{4} k_{1} \cdots d^{4} k_{m} \hat{I}_{\Gamma}\left(k, q, \alpha\left(\mathscr{E}_{\Gamma}\right), \alpha \beta\left(\mathscr{L}_{\Gamma}\right) ; \mu, a\right), \\
\hat{I}_{\Gamma}\left(k, q, \alpha\left(\mathscr{E}_{\Gamma}\right), \alpha \beta\left(\mathscr{L}_{\Gamma}\right) ; \mu, a\right)=\prod_{B \in \mathscr{B}_{\Gamma}} \hat{V}_{B}\left(\left\{l_{L}\right\}_{B},\left\{\alpha_{L}\right\}_{B} ; \mu, a\right) \cdot \prod_{L \in \mathscr{L}_{\Gamma}} \hat{\Delta}_{L}\left(l_{L}, \alpha_{L}, \beta_{L} ; \mu, a\right),
\end{gathered}
$$


where

$$
\begin{aligned}
\alpha\left(\mathscr{E}_{\Gamma}\right) & =\left(\alpha_{E} \mid E \in \mathscr{E}_{\Gamma}\right), & & \alpha_{E} \in \Lambda_{E} \quad \text { for all } E \in \mathscr{E}_{\Gamma}, \\
\alpha \beta\left(\mathscr{L}_{\Gamma}\right) & =\left(\left(\alpha_{L}, \beta_{L}\right) \mid L \in \mathscr{L}_{\Gamma}\right), & \alpha_{L} \in \Lambda_{L}^{1}, \beta_{L} \in \Lambda_{L}^{2} & \text { for all } L \in \mathscr{L}_{\Gamma} .
\end{aligned}
$$

Propagators are of the form

$$
\hat{\Delta}_{L}\left(l_{L}, \alpha_{L}, \beta_{L} ; \mu, a\right)=\frac{\hat{P}_{L}\left(l_{L} ; \mu, a\right)_{\alpha_{L} \beta_{L}}}{\prod_{j=1}^{n(L)}\left[e_{L j}\left(l_{L} ; a\right)+\mu_{L j}^{2}\right]},
$$

where $\alpha_{L} \in \Lambda_{L}^{1}, \beta_{L} \in \Lambda_{L}^{2}$, and for every pair $\left(\alpha_{L}, \beta_{L}\right)$ the numerator is of a form (2-12). Vertex functions $\hat{V}_{B}$ are also of a form (2-12), and $\left\{\alpha_{L}\right\}_{B}$ represents the indices of the line ends at the vertex B. Similarly, the integrand of a subdiagram $\gamma$ of $\Gamma$ is given by

$$
\hat{I}_{\gamma}\left(k^{\gamma}, q^{\gamma}, \alpha\left(\mathscr{E}_{\gamma}\right), \alpha \beta\left(\mathscr{L}_{\gamma}\right) ; \mu, a\right)=\prod_{B \in \mathscr{B}_{\gamma}} \hat{V}_{B}\left(\left\{l_{L}^{\gamma}\right\}_{B},\left\{\alpha_{L}^{\gamma}\right\}_{B} ; \mu, a\right) \cdot \prod_{L \in \mathscr{L}_{\gamma}} \hat{\Delta}_{L}\left(l_{L}^{\gamma}, \alpha_{L}, \beta_{L} ; \mu, a\right),
$$

where

$$
\alpha\left(\mathscr{E}_{\gamma}\right)=\left(\alpha_{E}^{\gamma} \mid E \in \mathscr{E}_{\gamma}\right), \quad \alpha \beta\left(\mathscr{L}_{\gamma}\right)=\left(\left(\alpha_{L}, \beta_{L}\right) \mid L \in \mathscr{L}_{\gamma}\right) .
$$

An "induced index" $\alpha_{E}^{\gamma}$ for $E \in \mathscr{E}_{\gamma}$ is equal to the index of the line ending of $L \in \mathscr{L}_{\Gamma}$ or $L \in \mathscr{E}_{\Gamma}$, which corresponds to $E$ by the imbedding of $\gamma$ as a subdiagram of $\Gamma$ (see Sect. 2). For $L \in \mathscr{L}_{\gamma}, \alpha_{L}^{\gamma}=\alpha_{L}$. Analogous statements can be made about reduced diagrams.

Internal momenta are defined as before. However, divergence degrees are modified to be independent of symmetry labels. For every 1PI subdiagram $\gamma$ of $\Gamma, \omega(\gamma)$ is defined as in (2-28). However, $\omega\left(\hat{\Delta}_{L}\right)$ and $\omega\left(\hat{V}_{B}\right)$ are now given by

$$
\begin{aligned}
& \omega\left(\hat{\Delta}_{L}\right)=\max _{\alpha_{L} \in \Lambda_{L}^{1}, \beta_{L} \in \Lambda_{L}^{2}} \overline{\operatorname{degr}}_{\hat{l}_{L}} \hat{\Delta}_{L}\left(l_{L}, \alpha_{L}, \beta_{L} ; \mu, a\right), \\
& \omega\left(\hat{V}_{B}\right)=\max _{\left\{\alpha_{L}\right\}_{B}} \overline{\operatorname{degr}}_{\left\{\hat{L}_{L}\right\}_{B}} \hat{V}_{B}\left(\left\{l_{L}\right\}_{B} ; \mu, a\right) .
\end{aligned}
$$

The same holds for reduced diagrams.

Finally, the forest formula is changed to

$$
\hat{\mathscr{R}}_{\Gamma}\left(q, \alpha\left(\mathscr{E}_{\Gamma}\right) ; \mu, a\right)=\sum_{\substack{\alpha_{L}, \beta_{L} \\ L \in \mathscr{L}_{\Gamma}}} \int_{-\pi / a}^{\pi / a} d^{4} k_{1} \cdots d^{4} k_{m} \hat{R}_{\Gamma}\left(k, q, \alpha\left(\mathscr{E}_{\Gamma}\right), \alpha \beta\left(\mathscr{L}_{\Gamma}\right) ; \mu, a\right),
$$

where

$$
\hat{R}_{\Gamma}\left(k, q, \alpha\left(\mathscr{E}_{\Gamma}\right), \alpha \beta\left(\mathscr{L}_{\Gamma}\right) ; \mu, a\right)=S_{\Gamma} \sum_{U \in \mathscr{W}} \prod_{\gamma \in U}\left(-\hat{t}_{\gamma}^{\delta(\gamma)} S_{\gamma}\right) \cdot \hat{I}_{\Gamma}(U),
$$

and the only restrictions to the subtraction degrees $\delta(\gamma)$ are given by (4-3) and (4-4). The convergence proof of (6-10) is identical to the above, the only modifications being that divergence degrees are now determined by (6-8) and (6-9). Finally, all comments we have made in Sect. 4 remain essentially unchanged.

A further generalization is to choose a subtraction point $\bar{q}$ different from zero. But all statements and calculations above are insensitive to such a choice. This is because the choice of normalization has no influence on the convergence properties 
of the Feynman integrals. A change of normalization conditions can be described by the addition of finite counterterms to the action which do not destroy renormalizability.

\section{Conclusions}

We have shown that the $\mathrm{BPHZ}$ renormalization procedure can be generalized in such a way that it applies to momentum-space Feynman integrals with a lattice cutoff. The generalization is that the counterterms, instead of being polynomials, are functions which are periodic with the Brillouin zone. They result from the wide class of lattice subtraction operators. This class includes as a special case polynomials in lattice momenta. In this case, after appropriate symmetrization in external momenta (as described in Sect. 4), they can be written as local counterterm contributions to the lattice action. Note that this symmetrization is necessary due to dependence of the counterterms on a chosen basis of external momenta.

The continuum limit of a massive lattice field theory which is renormalized in this way exists and is given by the field theory which is described by the (naive) $a \rightarrow 0$-limit of the lattice action, and which is renormalized by the BPHZ finite part prescription. This means that perturbation theory is universal, i.e., the continuum limit does not depend on the lattice action chosen. Also, the usual power counting renormalizability conditions of a field theory can be maintained, the only modification being that for all vertices of the theory the lattice UV-divergence degrees have to be less than or equal to four (in four-dimensional space-time). Especially, if all couplings are dimensionless, a lattice field theory is renormalizable if and only if its (formal) continuum limit is renormalizable (by power counting). Also, the choice of zero momentum as a subtraction point in the BPHZ procedure is of no importance. Any other choice is possible and corresponds to a change of the normalization conditions, which can be described by the addition of finite counterterms to the lattice action.

There are some general restrictions on the structure of Feynman integrals in momentum-space imposed by the renormalization procedure. In particular, the integrands have to be periodic with the Brillouin zone, a property which is reflected by the fact that the counterterms must also be periodic. In the formulation of the lattice power counting theorem [3] and the renormalization procedure for lattice Feynman diagrams, we have always assumed that the propagators have exactly one pole in the Brillouin zone, i.e. the denominator of every propagator takes its minimum at vanishing momentum only. Especially, lattice fermions with propagators having poles on the boundary of the Brillouin zone are excluded, whereas the renormalization procedure works e.g. for Wilson fermions. Furthermore, we have always assumed the numerator and denominator of the integrand to be $C^{\infty}$. This condition can be weakened in that the propagators should have this property at least in a small neighborhood of zero momentum, and globally they should be differentiable to such a degree that all differentiations necessary to subtract divergencies can be done without problems.

So far, we have discussed massive field theories in order to avoid infrared singularities. This allowed us to concentrate on the problems specific to the lattice 
as a UV-cutoff. If massless fields occur, we have to take into account possible infrared singularities very carefully. However, they are not specific to the lattice and are expected to be tractable by the methods which were developed for continuum Feynman integrals many years ago [6-9]. We will discuss this problem in a forthcoming paper and shall see that one only has to supplement the ultraviolet power counting conditions by infrared power counting conditions.

\section{Appendix A. Examples of Subtraction operators}

We give some general examples for $\hat{t}_{q}^{\delta}$ to be a subtraction operator. At first, we repeat the definition of the function classes $\mathscr{C}_{m}$ etc. of [3]. For $m \in \mathbf{Z}, \mathscr{C}_{m}$ is the class of functions $V$ of the form $V(u ; a)=F(u a) / a^{m}$, where $F \in C^{\infty}$. If in addition $\lim _{a \rightarrow 0} V(u ; a)$ exists, we get the class $\mathscr{C}_{m}^{c} . \mathscr{C}\left(\mathscr{C}^{c}\right)$ is the set of functions which are finite sums of functions in some $\mathscr{C}_{m}\left(\mathscr{C}_{m}^{c}\right)$. For $V(u, w ; a)=F(u a, w a) / a^{m} \in \mathscr{C}_{m}, r_{u}=$ $m-\overline{\operatorname{degr}}_{\hat{u}} V$ is the largest non-negative integer such that

$$
\left.\frac{\partial^{b} F(u, w)}{\partial w^{b}}\right|_{w=0} \equiv 0 \text { for }|b|<r_{u},
$$

where $|b|$ is the length of the multi-index $b$, i.e., the sum of its components. If $V=\sum_{i} V_{i} \in \mathscr{C}, V_{i} \in \mathscr{C}_{m_{i}}$ and $m_{i} \neq m_{k}$ for $i \neq k$, then $\overline{\operatorname{degr}}_{\hat{u}} V=\max _{i} \overline{\operatorname{degr}}_{\hat{u}} V_{i}$.

$\mathscr{F}$ is the set of all functions of the form $F=P / C$, where $P \in \mathscr{C}^{c}$ and the denominator $C \in \mathscr{C}^{c}$ is a finite product

$$
C=\prod_{i=1}^{n}\left[e_{i}\left(l_{i} ; a\right)+\mu_{i}^{2}\right] .
$$

$l_{i}$ are linear functions in momentum variables, and $e_{i}$ are functions as defined in (2-13). Note that

$$
\overline{\operatorname{degr}}_{\hat{u}}\left(e_{i}\left(l_{i} ; a\right)+\mu_{i}^{2}\right)= \begin{cases}2 & \text { if } l_{i} \text { depends on } u \\ 0 & \text { otherwise, }\end{cases}
$$

and this is equal to $\overline{\operatorname{degr}}_{u}\left(l_{i}^{2}+\mu_{i}^{2}\right)$ (see [1]). The UV-degree of $F$ with respect to $u$ is defined by

$$
\overline{\operatorname{degr}}_{\hat{u}} F \equiv \overline{\operatorname{degr}}_{\hat{u}} V-\overline{\operatorname{degr}}_{\hat{u}} C=\overline{\operatorname{degr}}_{\hat{u}} V-2 n_{u},
$$

where $n_{u}$ is the number of $l_{i}$ depending on $u$. For $F, F_{1}, F_{2} \in \mathscr{F}, \overline{\operatorname{degr}}_{\hat{u}}$ satisfies

$$
\begin{aligned}
& \overline{\operatorname{degr}}_{\hat{u}}\left(F_{1}+F_{2}\right) \leqq \max \left(\overline{\operatorname{degr}}_{\hat{u}} F_{1}, \overline{\operatorname{degr}}_{\hat{u}} F_{2}\right), \\
& \overline{\operatorname{degr}}_{\hat{u}}\left(F_{1} \cdot F_{2}\right) \leqq \overline{\operatorname{degr}}_{\hat{u}} F_{1}+\overline{\operatorname{degr}}_{\hat{u}} F_{2}, \\
& \overline{\operatorname{degr}}_{\hat{u}} \frac{\partial^{c}}{\partial u^{c}} F \leqq \overline{\operatorname{degr}}_{\hat{u}} F-|c|, \\
& \overline{\operatorname{degr}}_{\hat{u}} \frac{\partial^{c}}{\partial w^{c}} F \leqq \overline{\operatorname{degr}}_{\hat{u}} F .
\end{aligned}
$$

These are the "degree-properties" of [3], Lemma 2.2. 
Having reviewed general notions, we shall now state some general examples of subtraction operators. Let us introduce a special subset $\mathscr{M}$ of $\mathscr{C}^{c} . \mathscr{M}$ denotes the set of functions $P$ satisfying

$$
\begin{array}{r}
P(q ; a) \in \mathscr{C}^{c}, \\
\lim _{a \rightarrow 0} P(q ; a)=q,
\end{array}
$$

where $q$ is a real variable. If in addition $P$ is a finite product of $\sin$ and $\cos$ functions and is $(2 \pi / a)$-periodic in $q$, then $P$ is called a "lattice momentum". For instance, $[\sin (q a) / a]$ or $[(2 / a) \sin (q a / 2) \cos (q a / 2)]$ are lattice momenta.

For $\delta \in \mathbf{N}_{0}=\{0,1,2, \ldots\}$ consider the following construction: For every $j=1, \ldots, s$ and $i=\left(i_{1}, \ldots, i_{s}\right) \in \mathbf{N}_{0}^{s}, i_{1}+\cdots+i_{s} \leqq \delta$, let $P_{j \mid i}(q ; a) \in \mathscr{M}$ such that

Set

$$
\prod_{j=1}^{s} P_{j \mid i}\left(q_{j} ; a\right)^{i_{j}} \quad \text { is }(2 \pi / a) \text {-periodic in } q_{1}, \ldots, q_{s} .
$$

$$
\hat{t}_{q}^{\delta}=\sum_{n=0}^{\delta} \sum_{i_{1}, \ldots, i_{s} \in \mathbf{N}_{0}}^{i_{1}+\cdots+i_{s}=n} \frac{1}{i_{1} ! \cdots i_{s} !}\left[\prod_{j=1}^{s} P_{|l|}\left(q_{j} ; a\right)^{i_{j}}\right]\left[\frac{\partial^{i_{1}}}{\partial P_{1 \mid i}\left(q_{1} ; a\right)^{i_{1}}} \cdots \frac{\partial^{i_{s}}}{\partial P_{s \mid i}\left(q_{s} ; a\right)^{i_{s}}}\right]_{q=0} .
$$

This means that in application to a function $F$, which is $C^{\infty}$ in $q$, derivatives are taken at $q=0$.

Lemma A.1. If for every $j=1, \ldots, s$ and every $l \leqq \delta$ there exists a constant $c_{l j}$, so that

$$
\left(\frac{\partial^{l}}{\partial q^{l}} P_{j \mid i}(q ; a)\right)_{q=0}=c_{l j}
$$

for all $i$, then for arbitrary function $F$ which is $C^{\infty}$ in $q$

$$
\left[\frac{\partial^{n}}{\partial q^{n}}\left(1-\hat{t}_{q}^{\delta}\right) F\right]_{q=0}=0 \text { for all } n \in \mathbf{N}_{0}^{s},|n| \leqq \delta .
$$

This means that $\hat{t}_{q}^{\delta}$ is a subtraction operator of order $\delta$.

Proof. For a fixed $i \in \mathbf{N}_{0}^{s}, i_{1}+\cdots+i_{s} \leqq \delta$, choose

$$
P_{j}(q ; a) \equiv P_{j \mid i}(q ; a)
$$

for every $j=1, \ldots, s$. As a consequence of (A-2)

and

$$
\prod_{j=1}^{s} P_{j \mid i}\left(\lambda q_{j} ; a\right)^{i_{j}}=\prod_{j=1}^{s} P_{j}\left(\lambda q_{j} ; a\right)^{i_{j}}+O\left(\lambda^{\delta+1}\right)
$$

$$
\left(\frac{\partial^{i_{1}}}{\partial P_{1 \mid i}\left(q_{1} ; a\right)^{i_{1}}} \cdots \frac{\partial^{i_{s}}}{\partial P_{s \mid i}\left(q_{s} ; a\right)^{i_{s}}} F\right)_{q=0}=\left(\frac{\partial^{i_{1}}}{\partial P_{1}\left(q_{1} ; a\right)^{i_{1}}} \cdots \frac{\partial^{i_{s}}}{\partial P_{s}\left(q_{s} ; a\right)^{i_{s}}} F\right)_{q=0} .
$$

Hence, using Taylor's formula and that $P_{j} \in \mathscr{M}$, we get for $\lambda \rightarrow 0$,

$$
\left(1-\hat{t}_{\lambda q}^{\delta}\right) F=F-\sum_{n=0}^{\delta} \sum_{i_{1}, \ldots, i_{s} \in N_{0}}^{i_{1}+\cdots+i_{s}=n} \frac{1}{i_{1} ! \cdots i_{s} !}
$$




$$
\begin{aligned}
& \cdot\left[\prod_{j=1}^{s} P_{j}\left(\lambda q_{j} ; a\right)^{i_{j}}\right]\left(\frac{\partial^{i_{1}}}{\partial P_{1}\left(q_{1} ; a\right)^{i_{1}}} \cdots \frac{\partial^{i_{s}}}{\partial P_{s}\left(q_{s} ; a\right)^{i_{s}}} F\right)_{q=0}+O\left(\lambda^{\delta+1}\right) \\
& =O\left(\left[\sum_{j=1}^{s}\left|P_{j}\left(\lambda q_{j} ; a\right)\right|^{2}\right]^{(\delta+1) / 2}\right)+O\left(\lambda^{\delta+1}\right)=O\left(\lambda^{\delta+1}\right) . \quad \square
\end{aligned}
$$

As a corollary, we get

Lemma A.2. Let $P \in \mathscr{M}$. Then

$$
\hat{t}_{q}^{\delta}=\sum_{n=0}^{\delta} \frac{1}{n !} \sum_{i_{1}, \ldots, i_{n}=0}^{s} P\left(q_{i_{1}} ; a\right) \cdots P\left(q_{i_{n}} ; a\right)\left[\frac{\partial}{\partial P\left(q_{i_{1}} ; a\right)} \cdots \frac{\partial}{\partial P\left(q_{i_{n}} ; a\right)}\right]_{q=0}
$$

is a subtraction operator of order $\delta$.

As an example, set $P(q ; a)=\sin (q a) / a$. $P$ is a lattice momentum, and (A-7) is a subtraction operator. Note that $P$ is a periodic function. If the function class $\mathscr{F}$ is restricted appropriately, then we are allowed to use anti-periodic functions like $(2 / a) \sin (q a / 2)$ without violating (A-3). For instance, this can be done in the $\Phi^{4}$-theory as shown in Sect. 4.

\section{Appendix B. Chord Sets}

Lemma B.1. Let $\gamma$ be a connected Feynman diagram, $P$ the number of lines in $\gamma$ and $\mathscr{L}_{\gamma}=\left\{L_{1}, \ldots, L_{P}\right\}$. Define $\mathscr{T}_{\gamma}^{*} \subseteq \mathscr{L}_{\gamma}$ by

$$
L_{j} \in \mathscr{T}_{\gamma}^{*} \Leftrightarrow \mathscr{L}_{\gamma} \backslash\left\{L_{1}, \ldots, L_{j-1}\right\} \text { contains a loop } \mathscr{C} \supseteq\left\{L_{j}\right\},
$$

for every $j=1, \ldots, P$. Then $\mathscr{T}_{\gamma}^{*}$ is a chord set in $\gamma$.

Proof. We have to show that $\mathscr{T}_{\gamma}=\mathscr{L}_{\gamma} \backslash \mathscr{T}_{\gamma}^{*}$ is a tree in $\gamma$, i.e., $\mathscr{T}_{\gamma}$ contains no loop, but $\mathscr{T}_{\gamma} \cup\{L\}$ is not a tree, i.e., contains a loop, for every $L \in \mathscr{T}_{\gamma}^{*}$.

If $\mathscr{C} \subseteq \mathscr{T}_{\gamma}$ is a loop in $\mathscr{T}_{\gamma}$, then there exists a $k$ such that $L_{k} \in \mathscr{C} \subseteq \mathscr{L}_{\gamma} \backslash$ $\left\{L_{1}, \ldots, L_{k-1}\right\}$. But this is in contradiction to $L_{k} \in \mathscr{T}_{\gamma}$. Hence, $\mathscr{T}_{\gamma}$ contains no loop. To prove the second condition, let

$$
\mathscr{T}_{\gamma}^{*}=\left\{L_{i_{1}}, L_{i_{2}}, \ldots, L_{i_{m}}\right\} \subseteq \mathscr{L}_{\gamma}, \quad i_{1}<i_{2}<\cdots<i_{m} .
$$

We have to show that $\mathscr{T}_{\gamma} \cup\left\{L_{i_{k}}\right\}$ contains a loop, for every $k=1, \ldots, m$. If $L_{i_{k}}$ is a loop line, this is trivial. Let $B_{k}, C_{k} \in \mathscr{B}_{\gamma}$ be the endpoints of $L_{i_{k}}$. We show that there is a path $\mathscr{P}_{k} \subseteq \mathscr{T}_{\gamma}$ between $B_{k}$ and $C_{k}$. Then $\mathscr{P}_{k} \cup\left\{L_{i_{k}}\right\}$ is a loop in $\mathscr{T}_{\gamma} \cup\left\{L_{i_{k}}\right\}$.

If $k=m$, then by construction

$$
\mathscr{L}_{\gamma} \backslash\left\{L_{1}, \ldots, L_{i_{m-1}}\right\} \subseteq \mathscr{T}_{\gamma} \cup\left\{L_{i_{m}}\right\}
$$

contains a loop $\mathscr{C}_{m}$ such that $L_{i_{m}} \in \mathscr{C}_{m} . \mathscr{P}_{m}=\mathscr{C}_{m} \backslash\left\{L_{i_{m}}\right\} \subseteq \mathscr{T}_{\gamma}$ is a path between $B_{m}$ and $C_{m}$ in $\mathscr{T}_{\gamma}$. Assume the assertion holds for $k+1, \ldots, m$.

$$
\mathscr{L}_{\gamma} \backslash\left\{L_{1}, \ldots, L_{l_{k-1}}\right\}
$$

contains a loop $\mathscr{C}_{k}$, and $L_{i_{k}} \in \mathscr{C}_{k} . \mathscr{C}_{k} \backslash\left\{L_{i_{k}}\right\}$ is a path in $\mathscr{L}_{\gamma} \backslash\left\{L_{1}, \ldots, L_{i_{k-1}}\right\}$ between $B_{k}$ and $C_{k}$. Replacing for every $L_{i_{j}} \in \mathscr{C}_{k} \backslash\left\{L_{i_{k}}\right\}, j=k+1, \ldots, m,\left\{L_{i_{j}}\right\}$ by $\mathscr{P}_{j}$, the 
resulting set

$$
\mathscr{L}_{k}=\mathscr{C}_{k} \backslash\left\{L_{i_{k}}\right\} \backslash\left(\bigcup_{j=k+1}^{m}\left\{L_{i_{j}}\right\}\right) \bigcup_{j=k+1}^{m} \mathscr{P}_{j} \subseteq \mathscr{T}_{\gamma}
$$

is a connected set of lines in $\mathscr{T}_{\gamma}$. This set always contains a path $\mathscr{P}_{k}$ between $B_{k}$ and $C_{k}$.

\section{Appendix C. Complete Forests and the Dimension of Zimmermann Subspaces}

Let $\Gamma$ be a 1PI Feynman diagram, $H$ an arbitrary Zimmermann subspace, parametrized by $(t)=\left(t_{1}, \ldots, t_{h}\right)$ (and complementary parameters $\left.(v)\right)$, defined by (5-4), and $U$ a complete $\Gamma$-forest on $H$. For every $\gamma \in U$

$$
M_{U}(\gamma) \equiv 4 \sum_{\tau} m(\bar{\tau}(U))
$$

where the sum is over all $\tau \in U(\gamma) \cup\{\gamma\}, \bar{\tau}(U)$ variable on $H$, and $m(\bar{\tau}(U))$ is the number of loops in $\bar{\tau}(U)$. We prove that

$$
M_{U}(\Gamma) \geqq 4 h .
$$

Let $\mathscr{T}_{\Gamma}^{*}$ be a chord set in $\Gamma$. Every $k_{L}, L \in \mathscr{T}_{\Gamma}^{*}$, has a representation

$$
k_{L}(t, v, q)=\sum_{j=1}^{h} C_{L j} t_{j}+V_{L}(v, q)
$$

where $\operatorname{rank}\left(C_{L J}\right)=h$. For every $\mathscr{L} \subseteq \mathscr{T}_{\Gamma}^{*}$ we define

$$
\operatorname{rank}_{t} \mathscr{L}=\operatorname{rank}\left(C_{L j}\right)_{L \in \mathscr{L}},
$$

i.e., the rank of $C$ restricted to the rows $L \in \mathscr{L}$. $\operatorname{rank}_{t} \mathscr{L}$ is the maximal number of momenta $k_{L}, L \in \mathscr{L}$ which are linear independent with respect to $t$.

Lemma C.1. For every $\gamma \in U$

$$
M_{U}(\gamma) \geqq 4 \cdot \operatorname{rank}_{t} \mathscr{T}_{\gamma}^{*} .
$$

For $\Gamma$ this means $M_{U}(\Gamma) \geqq 4 \cdot \operatorname{rank}_{t} \mathscr{T}_{\Gamma}^{*}=4 h$.

Proof. By induction through the forest. For any $\mathscr{L} \subseteq \mathscr{L}_{\Gamma}$ let \# $\mathscr{L}$ be the number of lines in $\mathscr{L}$. First, let $\gamma$ be minimal in $U$. If $\gamma$ is constant, then $M_{U}(\gamma)=0$. But all lines of $\mathscr{T}_{\gamma}^{*}$ are constant, i.e., $\operatorname{rank}_{t} \mathscr{T}_{\gamma}^{*}=0$. If $\gamma$ is variable,

$$
M_{U}(\gamma)=4 m(\gamma)=4 \cdot \# \mathscr{T}_{\gamma}^{*} \geqq 4 \cdot \operatorname{rank}_{t} \mathscr{T}_{\gamma}^{*} .
$$

Next, let $\gamma \in U$ and $\gamma_{1}, \ldots, \gamma_{c}$ be the maximal elements of $U(\gamma)$, so that $\bar{\gamma}(U)=\gamma / \gamma_{1} \cdots \gamma_{c}$. By construction of chord sets in Sect. 2, $\mathscr{T}_{\gamma_{i}}^{*} \subseteq \mathscr{T}_{\gamma}^{*}$. Note that

$$
\mathscr{L}_{\gamma}=\mathscr{L}_{\gamma / \gamma_{1} \cdots \gamma_{c}} \bigcup_{i=1}^{c} \mathscr{T}_{\gamma_{i}} \bigcup_{i=1}^{c} \mathscr{T}_{\gamma_{i}}^{*} .
$$

The number of loops in $\bar{\gamma}(U)$ is given by

$$
\begin{aligned}
m(\bar{\gamma}(U)) & =m(\gamma)-\sum_{i=1}^{c} m\left(\gamma_{i}\right)=\# \mathscr{T}_{\gamma}^{*}-\sum_{i=1}^{c} \# \mathscr{T}_{\gamma_{i}}^{*} \\
& =\#\left(\mathscr{T}_{\gamma}^{*} \cap\left(\mathscr{L}_{\gamma / \gamma_{1} \cdot \gamma_{c}} \bigcup_{i=1}^{c} \mathscr{T}_{\gamma_{i}}\right)\right) .
\end{aligned}
$$

If $\bar{\gamma}(U)$ is variable, then 


$$
\begin{aligned}
M_{U}(\gamma) & =4 m(\bar{\gamma}(U))+\sum_{i=1}^{c} M_{U}\left(\gamma_{i}\right) \geqq 4 \cdot \#\left(\mathscr{T}_{\gamma}^{*} \cap\left(\mathscr{L}_{\gamma / \gamma_{1} \cdot \gamma_{c}} \bigcup_{i=1}^{c} \mathscr{T}_{\gamma_{t}}\right)\right)+4 \sum_{i=1}^{c} \operatorname{rank}_{t} \mathscr{T}_{\gamma_{i}}^{*} \\
& \geqq 4 \cdot \operatorname{rank}_{t}\left(\mathscr{T}_{\gamma}^{*} \cap\left(\mathscr{L}_{\gamma / \gamma_{1} \cdot \gamma_{c}} \bigcup_{i=1}^{c} \mathscr{T}_{\gamma_{\mathrm{t}}}\right)\right)+4 \sum_{i=1}^{c} \operatorname{rank}_{t} \mathscr{T}_{\gamma_{i}}^{*} \geqq 4 \cdot \operatorname{rank}_{t} \mathscr{T}_{\gamma}^{*},
\end{aligned}
$$

where we have used that for $\mathscr{A}, \mathscr{B} \subseteq \mathscr{T}_{\Gamma}^{*}: \operatorname{rank}_{t} \mathscr{A}+\operatorname{rank}_{t} \mathscr{B} \geqq \operatorname{rank}_{t}(\mathscr{A} \cup \mathscr{B})$. If $\bar{\gamma}(U)$ is constant, then

$$
M_{U}(\gamma)=\sum_{i=1}^{c} M_{U}\left(\gamma_{i}\right) \geqq 4 \sum_{i=1}^{c} \operatorname{rank}_{t} \mathscr{T}_{\gamma_{i}}^{*}
$$

a. For $L \in \mathscr{T}_{\gamma}^{*} \cap \mathscr{L}_{\gamma / \gamma_{1} \cdots \gamma_{c}}, k_{L}$ is constant, hence $\operatorname{rank}_{t}\left(\mathscr{T}_{\gamma}^{*} \cap \mathscr{L}_{\gamma / \gamma_{1} \cdots \gamma_{c}}\right)=0$.

b. For $L \in \mathscr{T}_{\gamma}^{*} \cap \mathscr{T}_{\gamma_{2}}$,

$$
k_{L}=k_{L}^{\gamma_{2}}+q_{L}^{\gamma_{2}}
$$

$L \notin \mathscr{T}_{\gamma}$, hence $q_{L}^{\gamma_{i}}$ is of the form

$$
q_{L}^{\gamma_{i}}=\sum_{E \in \mathscr{L}_{\gamma / \gamma_{1} \cdots \gamma_{c}}} d_{L E} k_{E}^{\gamma}=f_{L}(v, q),
$$

i.e., $q_{L}^{\gamma_{i}}$ is independent of $t$. Furthermore,

\section{Consequently}

$$
k_{L}^{\gamma_{i}}=\sum_{M \in \mathscr{T}_{\gamma_{t}}^{*}} c_{L M} k_{M}(t, v, q) .
$$

and

$$
k_{L}(t, v, q)=\sum_{M \in \mathscr{T}_{\gamma_{\mathrm{t}}}^{*}} c_{L M} k_{M}(t, v, q)+f_{L}(v, q),
$$

In summary

$$
\operatorname{rank}_{t} \mathscr{T}_{\gamma_{t}}^{*}=\operatorname{rank}_{t}\left(\mathscr{T}_{\gamma_{t}}^{*} \cup\left(\mathscr{T}_{\gamma}^{*} \cap \mathscr{T}_{\gamma_{i}}\right)\right)
$$

$$
\begin{aligned}
\sum_{i=1}^{c} \operatorname{rank}_{t} \mathscr{T}_{\gamma_{t}}^{*} & =\operatorname{rank}_{t}\left(\mathscr{T}_{\gamma}^{*} \cap \mathscr{L}_{\gamma / \gamma_{1} \ldots \gamma_{c}}\right)+\sum_{i=1}^{c} \operatorname{rank}_{t}\left(\mathscr{T}_{\gamma_{i}}^{*} \cup\left(\mathscr{T}_{\gamma}^{*} \cap \mathscr{T}_{\gamma_{i}}\right)\right) \\
& \geqq \operatorname{rank}_{t} \mathscr{T}_{\gamma}^{*} . \square
\end{aligned}
$$

Acknowledgement. I wish to thank M. Lüscher for many useful discussions.

\section{References}

1. Zimmermann, W.: Convergence of Bogoliubov's method of renormalization in momentum space. Commun. Math. Phys. 15, 208-234 (1969)

2. Hahn, Y., Zimmermann, W.: An elementary proof of Dyson's power counting theorem. Commun. Math. Phys. 10, 330-342 (1968)

3. Reisz, T.: A power counting theorem for Feynman integrals on the lattice. Commun. Math. Phys. 116, 81-126 (1988)

4. Gomes, M., Lowenstein, J. H., Zimmermann, W.: Generalization of the momentum space subtraction procedure for renormalized perturbation theory. Commun. Math. Phys. 39, 81-90 (1974)

5. Nakanishi, N.: Graph Theory and Feynman integrals. New York: Gordon and Breach 1971

6. Lowenstein, J. H.: Convergence theorems for renormalized Feynman integrals with Zero-Mass propagators. Commun. Math. Phys. 47, 53-68 (1976)

7. Lowenstein, J. H., Zimmermann, W.: The power counting theorem for Feynman integrals with massless propagators. Commun. Math. Phys. 44, 73-86 (1975) 
8. Bandelloni, G., Becchi, C., Blasi, A., Collina, R.: Renormalization of models with radiative mass generation. Commun. Math. Phys. 67, 147-178 (1978)

9. Lowenstein, J., Zimmermann, W.: On the formulation of theories with Zero-Mass propagators. Nucl. Phys. B86, 77 (1975)

Communicated by A. Jaffe

Received October 15, 1987 